\title{
Assessment of Contingent Liabilities for Risk Assets Evolutions Built on Brownian Motion
}

\author{
Nicholas Simon Gonchar \\ Bogolyubov Institute for Theoretical Physics of NAS of Ukraine, Kyiv, Ukraine \\ Email:mhonchar@i.ua
}

How to cite this paper: Gonchar, N.S (2020) Assessment of Contingent Liabilities for Risk Assets Evolutions Built on Brownian Motion. Advances in Pure Mathematics, 10, 259-296.

https://doi.org/10.4236/apm.2020.105016

Received: April 6, 2020

Accepted: May 9, 2020

Published: May 12, 2020

Copyright (C) 2020 by author(s) and Scientific Research Publishing Inc. This work is licensed under the Creative Commons Attribution International License (CC BY 4.0).

http://creativecommons.org/licenses/by/4.0/

(c) (i) Open Access

\begin{abstract}
This paper is a generalization of the results of the previous papers. Using these results a class of evolutions of risk assets based on the geometric Brownian motion is constructed. Among these evolutions of risk assets, the important class of the random processes is the random processes with parameters built on the basis of the discrete geometric Brownian motion. For this class of random processes the interval of non-arbitrage prices are found for the wide class of contingent liabilities. In particular, for the payoff functions of standard options call and put of the European type the fair prices of super-hedge are obtained. Analogous results are obtained for the put and call of arithmetical options of Asian type. For the parameters entering in the definition of random process the description of all statistical estimates is presented. Statistical estimate for which the fair price of super-hedge for the payoff functions of standard call and put options of European type is minimal is indicated. From the formulas found it follows that the fair price of super-hedge can be less than the price of the underlying asset. In terms of estimates the simple formula for the fair price of super-hedge is found. Every estimates can be realized in the reality. This depends on the distribution function of the observed dates in the financial market.
\end{abstract}

\section{Keywords}

Random Process, Regular Set of Measures, Optional Doob Decomposition, Local Regular Super-Martingale, Martingale, Assessment of Derivatives

\section{Introduction}

In reality, all financial markets are incomplete and the evolution of risky assets is discrete. The question arises, what random process describes the evolution of risky assets in the financial markets? This problem is important both from the perspective of the risk asset price behavior and from the risk hedging behavior of 
the risk asset.

In this work, which is a continuation of the paper [1], we construct the random processes based on the discrete geometric Brownian motion which can describe the evolution of risky assets. A new method of the description of martingale measures for the introduced class of evolutions of risk assets is developed. It is proved that every martingale measure can be represented as an integral on some measure on the set of extreme points of the set of martingale measures. This crucial fact is a base for the estimation of contingent liabilities in the incomplete financial markets with the evolution of risk assets introduced in [1]. The problem of estimation of the range of non arbitrage prices was began in the papers [2], [3] for the Levy exponential processes and the diffusion processes with jumps describing evolution of risk assets. The upper estimate for the standard call option payoff function in this paper coincides with the price of underlying asset. This fact is unacceptable from the economic point of view. In the proposed paper, we generalize the class of evolutions of risk assets proposed in [1] and which contains a class of evolutions built on the discrete geometric Brownian motion. For this class of evolutions of risk assets the set of martingale measures is described and the representation for every martingale measure as integral over the set of extreme points is obtained. Having this representation the formulas for the lower and upper bounds of non arbitrage prices are found. It is showed that the upper bound for the payoff functions of standard call option of European type is less than the price of underlying asset. The statistical estimates of parameters entering entering in the introduced evolutions of risk assets are obtained. The statistic for which the fair price of super-hedge is minimal is indicated.

In terms of statistical estimates the simple formulas for the fair price of super-hedge are obtained. Every estimate can be realized in the reality. This depends on distribution function of the observed dates in the financial market.

Assessment of risk in various systems was begun in papers [4] [5] [6] [7]. Construction of non-arbitrage model of evolution of risk assets see in [8] [9] [10] [11] [12]. Optional decomposition Theorems see in [13] [14] [15] [16].

\section{A Wide Class of Non-Arbitrage Evolutions of Risky Assets}

In this section, we generalize the results of the paper [1]. On the probability space $\left\{\Omega_{1}^{0}, \mathcal{F}_{1}^{0}, P_{1}^{0}\right\}$, let us consider the nonnegative random values $\xi_{i}\left(\omega_{1}\right), i=\overline{1, N}$, satisfying the conditions

$$
\begin{gathered}
0<P_{1}^{0}\left(\left\{\omega_{1} \in \Omega_{1}^{0}, \eta_{i}\left(\omega_{1}\right) \leq 0\right\}\right)<1, \\
0<P_{1}^{0}\left(\left\{\omega_{1} \in \Omega_{1}^{0}, \eta_{i}\left(\omega_{1}\right)>0\right\}\right), \quad i=\overline{1, N},
\end{gathered}
$$

where we introduced the denotation $\eta_{i}\left(\omega_{1}\right)=\xi_{i}\left(\omega_{1}\right)-1, i=\overline{1, N}$. Let $\{\Omega, \mathcal{F}\}$ be a direct product of the measurable spaces $\left\{\Omega_{i}^{0}, \mathcal{F}_{i}^{0}\right\}, i=\overline{1, N}$, where $\Omega_{i}^{0}=\Omega_{1}^{0}$, $\mathcal{F}_{i}^{0}=\mathcal{F}_{1}^{0}, \Omega=\prod_{i=1}^{N} \Omega_{i}^{0}$, and under the $\sigma$-algebra $\mathcal{F}$ we understand the minimal 
$\sigma$-algebra, generated by the sets $\prod_{i=1}^{N} G_{i}, G_{i} \in \mathcal{F}_{i}^{0}$. On the measurable space $\{\Omega, \mathcal{F}\}$, under the filtration $\mathcal{F}_{n}, n=\overline{1, N}$, we understand the minimal $\sigma$-algebra generated by the sets $\prod_{i=1}^{N} G_{i}, G_{i} \in \mathcal{F}_{i}^{0}$, where $G_{i}=\Omega_{i}^{0}$ for $i>n$. Further, we consider the probability space $\{\Omega, \mathcal{F}, P\}$, where $P=\prod_{i=1}^{N} P_{i}^{0}, P_{i}^{0}=P_{1}^{0}, i=\overline{1, N}$. Denote $\eta_{i}\left(\omega_{i}\right)=\xi_{i}\left(\omega_{i}\right)-1$ the random value which is given on the probability space $\left\{\Omega_{i}^{0}, \mathcal{F}_{i}^{0}, P_{i}^{0}\right\}$ and is distributed as $\eta_{i}\left(\omega_{1}\right)$ on the probability space $\left\{\Omega_{1}^{0}, \mathcal{F}_{1}^{0}, P_{1}^{0}\right\}$.

Described in Lemma 5 [1] the set of equivalent measures to the measure $P_{i}^{0}$ and such that $E^{Q} \eta_{i}\left(\omega_{i}\right)=0$, we denote by $M_{i}$.

On the measurable space $\{\Omega, \mathcal{F}\}$, we introduce into consideration the set of measures $M$, where $Q$ belongs to $M$, if $Q=\prod_{i=1}^{N} Q_{i}, Q_{i} \in M_{i}$. On the introduced measurable space $\{\Omega, \mathcal{F}, P\}$ let us consider the evolution of the risk asset given by the law

$$
S_{n}=S_{n-1}\left(1+a_{n}\left(\omega_{1}, \cdots, \omega_{n-1}\right) \eta_{n}\left(\omega_{n}\right)\right), \quad n=\overline{1, N},
$$

where the random values $a_{i}\left(\omega_{1}, \cdots, \omega_{i-1}\right)$ are $\mathcal{F}_{i-1}$-measurable, $i=\overline{1, N}$, satisfy the conditions $0<a_{i}\left(\omega_{1}, \cdots, \omega_{i-1}\right) \leq 1$. The main aim is to describe the set of martingale measures for the evolution of risk asset given by the formula (2). This problem we solved in Theorem 8 [1] in the case as the random values $\xi_{i}\left(\omega_{1}\right)=\xi_{1}\left(\omega_{1}\right), i=\overline{1, N}$.

Definition 1. Let $\left\{\Omega_{1}, \mathcal{F}_{1}\right\}$ be a measurable space. The decomposition $A_{n, k}, n, k=\overline{1, \infty}$, of the space $\Omega_{1}$ we call exhaustive one if the following conditions are valid:

1) $A_{n, k} \in \mathcal{F}_{1}, A_{n, k} \cap A_{n, s}=\varnothing, k \neq s, \bigcup_{k=1}^{\infty} A_{n, k}=\Omega_{1}, n=\overline{1, \infty}$;

2) the $(n+1)$-th decomposition is a sub-decomposition of the $n$-th one, that is, for every $j, A_{n+1, j} \subseteq A_{n, k}$ for a certain $k=k(j)$;

3) the minimal $\sigma$-algebra containing all $A_{n, k}, n, k=\overline{1, \infty}$, coincides with $\mathcal{F}_{1}$.

The next Remark 1 is important for the construction of the filtration having the exhaustive decomposition.

Remark 1. Suppose that the measurable spaces $\left\{\Omega_{1}, \mathcal{F}_{1}\right\}$ and $\left\{\Omega_{2}, \mathcal{F}_{2}\right\}$ have the exhaustive decompositions $A_{n, k}^{1}, n, k=\overline{1, \infty}$, and $A_{n, s}^{2}, m, s=\overline{1, \infty}$, respectively, then the measurable space $\left\{\Omega_{1} \times \Omega_{2}, \mathcal{F}_{1} \times \mathcal{F}_{2}\right\}$ also have the exhaustive decomposition $\quad B_{n, k s}, n=\overline{1, \infty}, k, s=\overline{1, \infty}, \quad B_{n, k s}=A_{n, k}^{1} \times A_{n, s}^{2}, k, s=\overline{1, \infty}, n=\overline{1, \infty}$. Really,

$$
\begin{aligned}
& \text { 1) } A_{n, k}^{1} \times A_{n, s}^{2} \in \mathcal{F}_{1} \times \mathcal{F}_{2}, A_{n, k}^{1} \times A_{n, s}^{2} \cap A_{n, t}^{1} \times A_{n, r}^{2}=\varnothing,(k, s) \neq(t, r), \\
& \bigcup_{k, s=1}^{\infty} B_{n, k s}=\Omega_{1} \times \Omega_{2}, n=\overline{1, \infty} ;
\end{aligned}
$$

2) the $(n+1)$-th decomposition is a sub-decomposition of the $n$-th one, that 
is, for every $k, s \quad B_{n+1, k s} \subseteq B_{n, i j}$ for a certain $i=i(k), j=j(s)$;

3) the minimal $\sigma$-algebra containing all $B_{n, k s}, n, k, s=\overline{1, \infty}$, coincides with $\mathcal{F}_{1} \times \mathcal{F}_{2}$.

In the next Lemma we give the sufficient condition of the existence of exhaustive decomposition. This Lemma is very important for the proof of the next Theorems [1].

Lemma 1. Let $\left\{\Omega_{1}, \mathcal{F}_{1}\right\}$ be a measurable space with a complete separable metric space $\Omega_{1}$ and Borel $\sigma$-algebra $\mathcal{F}_{1}$ on it. Then $\left\{\Omega_{1}, \mathcal{F}_{1}\right\}$ has an exhaustive decomposition.

Proof. If $\left\{\omega_{1}, \cdots, \omega_{n}, \cdots\right\}$ is a countable dense set in $\Omega_{1}$, then we denote

$$
B\left(\omega_{n}, \varepsilon_{m}\right)=\left\{\omega \in \Omega_{1}, \rho\left(\omega, \omega_{n}\right)<\varepsilon_{m}\right\}, \quad n, m=\overline{1, \infty},
$$

the countable set of open balls as $\varepsilon_{m}$ runs all positive rational numbers, where $\rho\left(\omega_{1}, \omega_{2}\right), \omega_{1}, \omega_{2} \in \Omega_{1}$ is a metric in $\Omega_{1}$. Prove that $\mathcal{F}_{1}=\sigma\left(B\left(\omega_{n}, \varepsilon_{m}\right), n, m=\overline{1, \infty}\right)$, where $\sigma\left(B\left(\omega_{n}, \varepsilon_{m}\right), n, m=\overline{1, \infty}\right)$ is a minimal $\sigma$-algebra generated by the sets (3). For this purpose let us prove that for every open set $A \in \Omega_{1}$ the representation

$$
A=\bigcup_{n_{k} \in N_{1}, m_{s} \in Q_{+}^{1}} B\left(\omega_{n_{k}}, \varepsilon_{m_{s}}\right)
$$

is true, where $N_{1}$ is a subset of positive integers, and $Q_{+}^{1}$ is a subset of positive rational numbers. Let us denote $\left\{\omega_{1}^{A}, \cdots, \omega_{n}^{A}, \cdots\right\}=A \cap\left\{\omega_{1}, \cdots, \omega_{n}, \cdots\right\}$. Suppose that $\omega_{0} \in A$, then $d=\inf _{\omega \in \bar{A} \backslash A} \rho\left(\omega_{0}, \omega\right)>0$, where $\bar{A}$ is a closure of the set $A$. Let the point $\omega_{k_{0}}^{A}$ belong to the ball $C\left(\omega_{0}, \frac{d}{8}\right)=\left\{\omega \in \Omega_{1}, \rho\left(\omega_{0}, \omega\right)<\frac{d}{8}\right\}$ and let us consider the ball

$C\left(\omega_{k_{0}}^{A}, \frac{d}{8}+\rho\left(\omega_{0}, \omega_{k_{0}}^{A}\right)\right)=\left\{\omega \in \Omega_{1}, \rho\left(\omega_{k_{0}}^{A}, \omega\right)<\frac{d}{8}+\rho\left(\omega_{0}, \omega_{k_{0}}^{A}\right)\right\}$. The point $\omega_{0}$ belongs to this ball and for every $\omega \in C\left(\omega_{k_{0}}^{A}, \frac{d}{8}+\rho\left(\omega_{0}, \omega_{k_{0}}^{A}\right)\right)$ the inequality

$$
\rho\left(\omega_{0}, \omega\right) \leq \rho\left(\omega_{0}, \omega_{k_{0}}^{A}\right)+\rho\left(\omega_{k_{0}}^{A}, \omega\right)<\frac{d}{8}+2 \rho\left(\omega_{0}, \omega_{k_{0}}^{A}\right)<\frac{3 d}{8}
$$

is true. Therefore $C\left(\omega_{k_{0}}^{A}, \frac{d}{8}+\rho\left(\omega_{0}, \omega_{k_{0}}^{A}\right)\right) \subset C\left(\omega_{0}, \frac{3 d}{8}\right)$. Let the rational number $\varepsilon_{k_{0}}$ satisfies the inequalities

$$
\frac{d}{8}+2 \rho\left(\omega_{0}, \omega_{k_{0}}^{A}\right)<\varepsilon_{k_{0}}<\frac{3 d}{8},
$$

then $C\left(\omega_{k_{0}}^{A}, \varepsilon_{k_{0}}\right) \subseteq C\left(\omega_{0}, \frac{d}{2}\right)$, since for every $\omega \in C\left(\omega_{k_{0}}^{A}, \varepsilon_{k_{0}}\right)$, $\rho\left(\omega_{0}, \omega\right) \leq \rho\left(\omega_{0}, \omega_{k_{0}}^{A}\right)+\rho\left(\omega_{k_{0}}^{A}, \omega\right)<\frac{d}{8}+\varepsilon_{k_{0}}<\frac{d}{2}$. So, for $\omega_{0} \in A$ we found $\omega_{k_{0}}^{A} \in\left\{\omega_{1}, \cdots, \omega_{n}, \cdots\right\}$ and the rational number $\varepsilon_{k_{0}}$ such that 
$\omega_{0} \in C\left(\omega_{k_{0}}^{A}, \varepsilon_{k_{0}}\right) \subset C\left(\omega_{0}, \frac{d}{2}\right) \subset A$. The last prove the needed statement. To complete the proof of Lemma 1 let us construct the exhaustive decomposition. Let us renumber the sets $B\left(\omega_{n}, \varepsilon_{m}\right)$ putting by $D_{1}=B\left(\omega_{1}, \varepsilon_{1}\right), D_{2}=B\left(\omega_{1}, \varepsilon_{2}\right)$, $D_{3}=B\left(\omega_{2}, \varepsilon_{1}\right)$, and so on. We put that $\left\{A_{1 k}\right\}_{k=1}^{\infty}$ consists of two sets $D_{1}$ and $\bar{D}_{1}=\Omega_{1} \backslash D_{1}$. If the set $\left\{A_{n k}\right\}_{k=1}^{\infty}$ is constructed, then the set $\left\{A_{n+1 k}\right\}_{k=1}^{\infty}$ we construct from the various set of the kind $A_{n k} \cap D_{n+1}, A_{n k} \cap \bar{D}_{n+1}$. By construction the minimal $\sigma$-algebra $\sigma\left\{A_{n k}, n, k=\overline{1, \infty}\right\}=\sigma\left\{B\left(\omega_{n}, \varepsilon_{m}\right), n, m=\overline{1, \infty}\right\}$. Taking into account the previous part of the proof we have $\sigma\left\{A_{n k}, n, k=\overline{1, \infty}\right\}=F_{1}$. Lemma 1 is proved.

Below, we describe completely the regular set of measures, introduced in [1], in the case as $\xi_{0}=\prod_{i=1}^{N}\left[1+a_{i}\left(\omega_{1}, \cdots, \omega_{i-1}\right) \eta_{i}\left(\omega_{i}\right)\right], \quad N<\infty, \quad 0<a_{i}\left(\omega_{1}, \cdots, \omega_{i-1}\right) \leq 1$, $i=\overline{1, N}$, and the random values $\xi_{i}\left(\omega_{1}\right), i=\overline{1, N}$, are integrable ones relative to the measure $P_{1}^{0}$. For this purpose, we introduce the denotations:

$\Omega_{i}^{-}=\left\{\omega_{i} \in \Omega_{i}^{0}, \eta_{i}\left(\omega_{i}\right) \leq 0\right\}, \Omega_{i}^{+}=\left\{\omega_{i} \in \Omega_{i}^{0}, \eta_{i}\left(\omega_{i}\right)>0\right\}, P_{i}^{-}$is a contraction of the measure $P_{i}^{0}$ on the $\sigma$-algebra $\mathcal{F}_{i}^{-}, P_{i}^{+}$is a contraction of the measure $P_{i}^{0}$ on the $\sigma$-algebra $\mathcal{F}_{i}^{+}, \mathcal{F}_{i}^{-}=\Omega_{i}^{-} \cap \mathcal{F}_{i}^{0}, \mathcal{F}_{i}^{+}=\Omega_{i}^{+} \cap \mathcal{F}_{i}^{0}$. Denote $U_{i}=\Omega_{i}^{-} \times \Omega_{i}^{+}$ and introduce the measure $\mu_{i}=P_{i}^{-} \times P_{i}^{+}$on the $\sigma$-algebra $\mathcal{G}_{i}=\mathcal{F}_{i}^{-} \times \mathcal{F}_{i}^{+}$. Let us introduce the measurable space $\{\mathcal{V}, \mathcal{L}, \mu\}$, where $\mathcal{V}=\prod_{i=1}^{N} U_{i}$, is a direct product of the spaces $U_{i}=\Omega_{i}^{-} \times \Omega_{i}^{+}, \quad i=\overline{1, N}, \mathcal{L}=\prod_{i=1}^{N} \mathcal{G}_{i}$ is a direct product of the $\sigma$ algebras $\mathcal{G}_{i}, \quad i=\overline{1, N}$. At last, let $\mu=\prod_{i=1}^{N} \mu_{i}$ be a direct product of the measures $\mu_{i}, \quad i=\overline{1, N}$, and let $v_{v}=\prod_{i=1}^{N} v_{\omega_{i}^{1}, \omega_{i}^{2}}, \quad v=\left\{\left(\omega_{1}^{1}, \omega_{1}^{2}\right), \cdots,\left(\omega_{N}^{1}, \omega_{N}^{2}\right)\right\}$, be a direct product of the measures $v_{\omega_{1}^{1}, \omega_{1}^{2}}, i=\overline{1, N}$, which is a countable additive function on the $\sigma$-algebra $\mathcal{F}_{N}$ for every $v \in \mathcal{V}$, where

$$
v_{\omega_{i}^{1}, \omega_{i}^{2}}\left(A_{i}\right)=\chi_{A_{i}}\left(\omega_{i}^{1}\right) \frac{\eta_{i}^{+}\left(\omega_{i}^{2}\right)}{\eta_{i}^{-}\left(\omega_{i}^{1}\right)+\eta_{i}^{+}\left(\omega_{i}^{2}\right)}+\chi_{A_{i}}\left(\omega_{i}^{2}\right) \frac{\eta_{i}^{-}\left(\omega_{i}^{1}\right)}{\eta_{i}^{-}\left(\omega_{i}^{1}\right)+\eta_{i}^{+}\left(\omega_{i}^{2}\right)}
$$

for $\omega_{i}^{1} \in \Omega_{i}^{-}, \omega_{i}^{2} \in \Omega_{i}^{+}, \quad A_{i} \in \mathcal{F}_{i}^{0}$.

In the next Theorem 1 , we assume that the random values $\eta_{i}\left(\omega_{i}\right), i=\overline{1, N}$, are integrable ones.

Theorem 1. On the measurable space $\{\Omega, \mathcal{F}\}$ with the filtration $\mathcal{F}_{N}$ on it, every measure $Q$ of the regular set of measures $M$ for the random value

$\xi_{0}=\prod_{i=1}^{N}\left[1+a_{i}\left(\omega_{1}, \cdots, \omega_{i-1}\right) \eta_{i}\left(\omega_{i}\right)\right], \quad N<\infty, 0<a_{i}\left(\omega_{1}, \cdots, \omega_{i-1}\right) \leq 1, \quad i=\overline{1, N}$, has the representation

$$
Q(A)=\int_{\mathcal{V}} \alpha(v) v_{v}(A) \mathrm{d} \mu(v)
$$


where the random value $\alpha(v)$ satisfies the conditions

$$
\begin{gathered}
\mu(\{v \in \mathcal{V}, \alpha(v)>0\})=\prod_{i=1}^{N} P_{i}^{0}\left(\Omega_{i}^{-}\right) P_{i}^{0}\left(\Omega_{i}^{+}\right), \\
\int_{\mathcal{V}} \alpha(v) \prod_{i=1}^{N} \frac{\eta_{i}^{-}\left(\omega_{i}^{1}\right) \eta_{i}^{+}\left(\omega_{i}^{2}\right)}{\eta_{i}^{-}\left(\omega_{i}^{1}\right)+\eta_{i}^{+}\left(\omega_{i}^{2}\right)} \mathrm{d} \mu(v)<\infty, \\
\int_{\mathcal{V}} \alpha(v) \mathrm{d} \mu(v)=1 .
\end{gathered}
$$

Proof. To prove Theorem, it needs to prove that the countable additive measure $v_{v}(A)$ at every fixed $v \in \mathcal{V}$ is a measurable map from the measurable space $\{\mathcal{V}, \mathcal{L}\}$ into the measurable space $\{[0,1], B([0,1])\}$ for every fixed $A \in \mathcal{F}_{N}$. For $A=\prod_{i=1}^{N} A_{i}, \quad A_{i} \in \mathcal{F}_{i}^{0}, \quad v_{v}(A)$ is a measurable map from the measurable space $\{\mathcal{V}, \mathcal{L}\}$ into the measurable space $\{[0,1], B([0,1])\}$. The family of sets of the kind $\bigcup_{i \in I} E_{i}, \quad E_{i}=\prod_{s=1}^{N} A_{s}^{i}, \quad A_{s}^{i} \in \mathcal{F}_{s}^{0}$, where $E_{i} \cap E_{j}=\varnothing$, the set $I$ is an arbitrary finite set, forms the algebra of the sets that we denote by $U_{0}$. From the countable additivity of $v_{v}(A), v_{v}\left(\bigcup_{i \in I} E_{i}\right)=\sum_{i \in I} v_{v}\left(E_{i}\right)$ is a measurable map from the measurable space $\{\mathcal{V}, \mathcal{L}\}$ into the measurable space $\{[0,1], B([0,1])\}$. Let $T$ be a class of the sets from the minimal $\sigma$-algebra $\Sigma$ generated by $U_{0}$ for every subset $E$ of that $v_{v}(E)$ is a measurable map from the measurable space $\{\mathcal{V}, \mathcal{L}\}$ into the measurable space $\{[0,1], B([0,1])\}$. Let us prove that $T$ is a monotonic class. Suppose that $E_{i} \subset E_{i+1}, i=\overline{1, \infty}, E_{i} \in T$. Then, $v_{v}\left(E_{i}\right) \leq v_{v}\left(E_{i+1}\right)$. From this, it follows that $\lim _{i \rightarrow \infty} v_{v}\left(E_{i}\right)$ is a measurable map from the measurable space $\{\mathcal{V}, \mathcal{L}\}$ into the measurable space $\{[0,1], B([0,1])\}$. But, $v_{v}\left(E_{i+1} \backslash E_{i}\right)=v_{v}\left(E_{i+1}\right)-v_{v}\left(E_{i}\right)$ is a measurable map from $\{\mathcal{V}, \mathcal{L}\}$ into $\{[0,1], B([0,1])\}$. From this equality, it follows that the set $E_{i+1} \backslash E_{i}$ belongs to the class $T$. Since $\bigcup_{i=1}^{\infty} E_{i}=E_{1} \cup \bigcup_{i=1}^{\infty}\left[E_{i+1} \backslash E_{i}\right]$, we have

$$
\begin{aligned}
\lim _{n \rightarrow \infty} v_{v}\left(E_{n}\right) & =v_{v}\left(E_{1}\right)+\lim _{n \rightarrow \infty} \sum_{i=1}^{n} v_{v}\left(E_{i+1} \backslash E_{i}\right) \\
& =v_{v}\left(E_{1}\right)+\sum_{i=1}^{\infty} v_{v}\left(E_{i+1} \backslash E_{i}\right) \\
& =v_{v}\left(E_{1} \cup \bigcup_{i=1}^{\infty}\left[E_{i+1} \backslash E_{i}\right]\right)=v_{v}\left(\bigcup_{i=1}^{\infty} E_{i}\right) .
\end{aligned}
$$

The equalities (12) mean that $\bigcup_{i=1}^{\infty} E_{i}$ belongs to $T$, since $v_{v}\left(\bigcup_{i=1}^{\infty} E_{i}\right)$ is a measurable map of $\{\mathcal{V}, \mathcal{L}\}$ into $\{[0,1], B([0,1])\}$. Suppose that $E_{i} \supset E_{i+1}, E_{i} \in T$, $i=\overline{1, \infty}$. Then, this case is reduced to the previous one by the note that the sequence $\bar{E}_{i}=\prod_{i=1}^{N} \Omega_{i}^{0} \backslash E_{i}, i=\overline{1, \infty}$, is monotonically increasing. From this, it follows 
that $\bar{E}=\bigcup_{i=1}^{\infty} \bar{E}_{i} \in T$. Therefore, $\bigcap_{i=1}^{\infty} E_{i}=\prod_{i=1}^{N} \Omega_{i}^{0} \backslash \bigcup_{i=1}^{\infty} \bar{E}_{i} \in T$. Thus, $T$ is a monotone class. But, $U_{0} \subset T$. Hence, $T$ contains the minimal monotone class generated by the algebra $U_{0}$, that is, $m\left(U_{0}\right)=\Sigma$, therefore, $\Sigma \subset T$. Thus, $v_{v}(E)$ is a measurable map of $\{\mathcal{V}, \mathcal{L}\}$ into $\{[0,1], B([0,1])\}$ for $A \in \Sigma$. The fact that the random value $\alpha(v)$ satisfies the conditions (9)-(11) means that $Q$, given by the formula (8), is a countable additive function of sets and $E^{Q} \xi_{0}<\infty$. Moreover, $E^{Q} \xi_{0}=1$. It is evident that $E^{Q}\left\{\xi_{0} \mid \mathcal{F}_{n}\right\}=\prod_{i=1}^{n}\left[1+a_{i}\left(\omega_{1}, \cdots, \omega_{i-1}\right) \eta_{i}\left(\omega_{i}\right)\right], Q \in M$.

Due to Lemma 4, [1], this proves that the set $M$ is a regular set of measure. Theorem 1 is proved.

Remark 2. The representation (8) for the regular set of measures $M$ means that $M$ is a convex set of equivalent measures. Since the random value $\alpha(v)$ runs all bounded random values, satisfying the conditions $(9-11)$, it is easy to show that the set of measures $v_{v}(A), v \in \mathcal{V}, A \in \mathcal{F}_{N}$, is the set of extreme points for the set $M$.

Let us introduce the denotations (see also [1])

$$
\begin{gathered}
m_{n}(\{\omega\})=\prod_{i=1}^{n}\left[1+a_{i}\left(\omega_{1}, \cdots, \omega_{i-1}\right) \eta_{i}\left(\omega_{i}\right)\right], 1 \leq n \leq N<\infty, \\
0<a_{i}\left(\omega_{1}, \cdots, \omega_{i-1}\right) \leq 1, \quad i=\overline{1, N} . \\
\{\omega\}=\left\{\omega_{1}, \cdots, \omega_{N}\right\}, \quad\{\omega\}^{i}=\left\{\omega_{1}^{i}, \cdots, \omega_{N}^{i}\right\}, \quad i=1,2, \quad \Omega_{n}=\prod_{i=1}^{n} \Omega_{i}^{0}, \quad n=\overline{1, N}, \\
\{\omega\}_{n}=\left\{\omega_{1}, \cdots, \omega_{n}\right\}, \quad\{\omega\}_{n}^{i}=\left\{\omega_{1}^{i}, \cdots, \omega_{n}^{i}\right\}, \quad i=1,2, \\
\bar{\Omega}_{n}^{-}=\left\{\{\omega\}_{n} \in \Omega_{n},\left[m_{n}-m_{n-1}\right]\left(\{\omega\}_{n}\right) \leq 0\right\}=\Omega_{n-1} \times\left\{\omega_{n} \in \Omega_{n}^{0}, \eta_{n}\left(\omega_{n}\right) \leq 0\right\}, \\
\bar{\Omega}_{n}^{+}=\left\{\{\omega\}_{n} \in \Omega_{n},\left[m_{n}-m_{n-1}\right]\left(\{\omega\}_{n}\right)>0\right\}=\Omega_{n-1} \times\left\{\omega_{n} \in \Omega_{n}^{0}, \eta_{n}\left(\omega_{n}\right)>0\right\}, \\
\Omega_{n}^{-}=\left\{\{\omega\} \in \Omega_{N},\left[m_{n}-m_{n-1}\right](\{\omega\}) \leq 0\right\}=\Omega_{n-1} \times\left\{\omega_{n} \in \Omega_{n}^{0}, \eta_{n}\left(\omega_{n}\right) \leq 0\right\} \times \Omega_{N-n}, \\
\Omega_{n}^{+}=\left\{\{\omega\} \in \Omega_{N},\left[m_{n}-m_{n-1}\right](\{\omega\})>0\right\} \\
=\Omega_{n-1} \times\left\{\omega_{n} \in \Omega_{n}^{0}, \eta_{n}\left(\omega_{n}\right)>0\right\} \times \Omega_{N-n}, \quad n=\overline{1, N},
\end{gathered}
$$

Note that the $\sigma$-algebra $\mathcal{F}_{n}$ is generated by sets of the kind $G=\prod_{i=1}^{N} G_{i}$, where $G_{i} \in \mathcal{F}_{i}^{0}, \quad i=\overline{1, n}, G_{i}=\Omega_{i}^{0}, \quad i=\overline{n+1, N}$. Denote $P_{n}=\prod_{i=1}^{n} P_{i}^{0}$ the contraction of the measure $P_{N}=\prod_{i=1}^{N} P_{i}^{0}$ onto the $\sigma$-algebra $\mathcal{F}_{n}$. Further we use the denotations $P_{n}^{-}$and $P_{n}^{+}$which are the contractions the measure $P_{n}$ onto the $\sigma$-algebras $\mathcal{F}_{n} \cap \Omega_{n}^{-}$and $\mathcal{F}_{n} \cap \Omega_{n}^{+}$, correspondingly. If the measure $Q$ belongs to the set of martingale measures (8), then $E^{Q}\left\{m_{n} \mid \mathcal{F}_{n-1}\right\}=m_{n-1}$, or $E^{Q}\left[m_{n}-m_{n-1}\right]=0$. From this, for the measure $Q$ the representation 


$$
\begin{aligned}
& Q\left(A_{1}\right)=\int_{\Omega_{n}^{-} \times \Omega_{n}^{+}} \frac{\chi_{A_{1}}\left(\{\omega\}^{1}\right) \alpha\left(\{\omega\}^{1} ;\{\omega\}^{2}\right)\left[m_{n}-m_{n-1}\right]^{+}\left(\{\omega\}^{2}\right)}{\left[m_{n}-m_{n-1}\right]^{-}\left(\{\omega\}^{1}\right)+\left[m_{n}-m_{n-1}\right]^{+}\left(\{\omega\}^{2}\right)} \mathrm{d}\left[P_{N}^{-} \times P_{N}^{+}\right] \\
& +\int_{\Omega_{n}^{-} \times \Omega_{n}^{+}} \frac{\chi_{A_{1}}\left(\{\omega\}^{2}\right) \alpha\left(\{\omega\}^{1} ;\{\omega\}^{2}\right)\left[m_{n}-m_{n-1}\right]^{-}\left(\{\omega\}^{1}\right)}{\left[m_{n}-m_{n-1}\right]^{-}\left(\{\omega\}^{1}\right)+\left[m_{n}-m_{n-1}\right]^{+}\left(\{\omega\}^{2}\right)} \mathrm{d}\left[P_{N}^{-} \times P_{N}^{+}\right], A_{1} \in \mathcal{F}_{n},
\end{aligned}
$$

is true if the random value $\alpha\left(\{\omega\}^{1} ;\{\omega\}^{2}\right)>0$ satisfies the condition

$$
\int_{\Omega_{n}^{-} \times \Omega_{n}^{+}} \alpha\left(\{\omega\}^{1} ;\{\omega\}^{2}\right) \mathrm{d}\left[P_{N}^{-} \times P_{N}^{+}\right]=1 .
$$

Since for the set $A_{1}$ the representation $A_{1}=A \times \prod_{i=n+1}^{N} \Omega_{i}^{0}$, is true, where $A \in \overline{\mathcal{F}}_{n}=\prod_{i=1}^{n} \mathcal{F}_{i}^{0}$, then for the contraction $Q_{n}$ of the measure $Q$ onto the $\sigma$-algebra $\mathcal{F}_{n}$ the representation

$$
\begin{aligned}
& Q_{n}(A)=\int_{\bar{\Omega}_{n}^{-} \times \bar{\Omega}_{n}^{+}} \frac{\chi_{A}\left(\{\omega\}_{n}^{1}\right) \alpha_{n}^{1}\left(\{\omega\}_{n}^{1} ;\{\omega\}_{n}^{2}\right)\left[m_{n}-m_{n-1}\right]^{+}\left(\{\omega\}_{n}^{2}\right)}{\left[m_{n}-m_{n-1}\right]^{-}\left(\{\omega\}_{n}^{1}\right)+\left[m_{n}-m_{n-1}\right]^{+}\left(\{\omega\}_{n}^{2}\right)} \mathrm{d}\left[\bar{P}_{n}^{-} \times \bar{P}_{n}^{+}\right] \\
& +\int_{\bar{\Omega}_{n}^{-} \times \bar{\Omega}_{n}^{+}} \frac{\chi_{A}\left(\{\omega\}_{n}^{2}\right) \alpha_{n}^{1}\left(\{\omega\}_{n}^{1} ;\{\omega\}_{n}^{2}\right)\left[m_{n}-m_{n-1}\right]^{-}\left(\{\omega\}_{n}^{1}\right)}{\left[m_{n}-m_{n-1}\right]^{-}\left(\{\omega\}_{n}^{1}\right)+\left[m_{n}-m_{n-1}\right]^{+}\left(\{\omega\}_{n}^{2}\right)} \mathrm{d}\left[\bar{P}_{n}^{-} \times \bar{P}_{n}^{+}\right], \quad A \in \overline{\mathcal{F}}_{n},
\end{aligned}
$$

is true, where we introduced the denotations $\bar{P}_{n}^{-}$and $\bar{P}_{n}^{+}$which are the contractions of the measure $P_{n}$ onto the $\sigma$-algebras $\overline{\mathcal{F}}_{n} \cap \bar{\Omega}_{n}^{-}$and $\overline{\mathcal{F}}_{n} \cap \bar{\Omega}_{n}^{+}$, correspondingly,

$$
\begin{gathered}
\alpha_{n}^{1}\left(\{\omega\}_{n}^{1} ;\{\omega\}_{n}^{2}\right)=\int_{\Omega_{N-n} \times \Omega_{N-n}} \alpha\left(\{\omega\}^{1} ;\{\omega\}^{2}\right) \mathrm{d}\left[P_{N-n} \times P_{N-n}\right], \\
P_{N-n}=\prod_{i=n+1}^{N} P_{i}^{0}, \quad \int_{\bar{\Omega}_{n}^{-} \times \Omega_{n}^{+}} \alpha_{n}^{1}\left(\{\omega\}_{n}^{1} ;\{\omega\}_{n}^{2}\right) \mathrm{d}\left[\bar{P}_{n}^{-} \times \bar{P}_{n}^{+}\right]=1 .
\end{gathered}
$$

In the set $\Omega_{n}^{-} \times \Omega_{n}^{+}$let us introduce the transformation

$$
\begin{gathered}
T_{n}\left(\{\omega\}_{n}^{1} ;\{\omega\}_{n}^{2}\right)=\left(T_{n}^{1}\left(\{\omega\}_{n}^{1}\right) ; T_{n}^{2}\left(\{\omega\}_{n}^{2}\right)\right), \\
T_{n}^{1}\left(\{\omega\}_{n}^{1}\right)=\left\{\{\omega\}_{n-1}^{2}, \omega_{n}^{1}\right\}, \quad T_{n}^{2}\left(\{\omega\}_{n}^{2}\right)=\left\{\{\omega\}_{n-1}^{1}, \omega_{n}^{2}\right\}, \quad n=\overline{1, N} .
\end{gathered}
$$

By the definition we put that for $n=1$ the transformation $T_{1}$ is identical one. Introduce the denotations

$$
\begin{gathered}
v_{n}^{1}\left(\{\omega\}_{n}^{1} ;\{\omega\}_{n}^{2}\right)=\frac{\left[m_{n}-m_{n-1}\right]^{+}\left(\{\omega\}_{n}^{2}\right)}{\varphi_{n}\left(\{\omega\}_{n}^{1} ;\{\omega\}_{n}^{2}\right)}, \\
v_{n}^{2}\left(\{\omega\}_{n}^{1} ;\{\omega\}_{n}^{2}\right)=\frac{\left[m_{n}-m_{n-1}\right]^{-}\left(\{\omega\}_{n}^{1}\right)}{\varphi_{n}\left(\{\omega\}_{n}^{1} ;\{\omega\}_{n}^{2}\right)} . \\
\varphi_{n}^{1}\left(\{\omega\}_{n}^{1} ;\{\omega\}_{n}^{2}\right)=\left[m_{n}-m_{n-1}\right]^{-}\left(\{\omega\}_{n}^{1}\right)+\left[m_{n}-m_{n-1}\right]^{+}\left(\{\omega\}_{n}^{2}\right),
\end{gathered}
$$




$$
\varphi_{n}\left(\{\omega\}_{n}^{1} ;\{\omega\}_{n}^{2}\right)=\varphi_{n}^{1}\left(\{\omega\}_{n}^{1} ;\{\omega\}_{n}^{2}\right)+\varphi_{n}^{1}\left(T_{n}\left(\left(\{\omega\}_{n}^{1} ;\{\omega\}_{n}^{2}\right)\right)\right) .
$$

Theorem 2. Let $\Omega_{1}^{0}$ be a complete separable metric space and $\mathcal{F}_{1}^{0}$ be a Borel $\sigma$-algebra on it. If the condition

$$
\int_{\Omega_{n}} f\left(\{\omega\}_{n}\right) \mathrm{d} P_{n}<\infty
$$

is true for $\mathcal{F}_{n}$-measurable nonnegative random value $f\left(\{\omega\}_{n}\right)$, then the closure of the set of points $E^{Q_{n}} f\left(\{\omega\}_{n}\right), Q_{n} \in M_{n}$, in metrics $\rho(x, y)=|x-y|$ on the real line contains the set of points

$$
\begin{aligned}
& f\left(\{\omega\}_{n}^{1}\right) v_{n}^{1}\left(\{\omega\}_{n}^{1} ;\{\omega\}_{n}^{2}\right)+f\left(\{\omega\}_{n}^{2}\right) v_{n}^{2}\left(\{\omega\}_{n}^{1} ;\{\omega\}_{n}^{2}\right) \\
& +f\left(T_{n}^{1}\left(\{\omega\}_{n}^{1}\right)\right) v_{n}^{1}\left(T_{n}\left(\{\omega\}_{n}^{1} ;\{\omega\}_{n}^{2}\right)\right) \\
& +f\left(T_{n}^{2}\left(\{\omega\}_{n}^{2}\right)\right) v_{n}^{2}\left(T_{n}\left(\{\omega\}_{n}^{1} ;\{\omega\}_{n}^{2}\right)\right), n=\overline{1, N} .
\end{aligned}
$$

Proof. Let us find the conditions for the measurable functions $\alpha_{n}^{1}\left(\{\omega\}_{n}^{1} ;\{\omega\}_{n}^{2}\right)$ under which $E^{Q_{n}}\left\{m_{n} \mid \overline{\mathcal{F}}_{n-1}\right\}=m_{n-1}$. Introduce the denotation

$$
\alpha_{n}^{0}\left(\{\omega\}_{n}^{1} ;\{\omega\}_{n}^{2}\right)=\frac{\alpha_{n}^{1}\left(\{\omega\}_{n}^{1} ;\{\omega\}_{n}^{2}\right)}{\left[m_{n}-m_{n-1}\right]^{-}\left(\{\omega\}_{n}^{1}\right)+\left[m_{n}-m_{n-1}\right]^{+}\left(\{\omega\}_{n}^{2}\right)} .
$$

Let the set $B$ belongs to $\overline{\mathcal{F}}_{n-1}$, then

$$
\begin{aligned}
& E^{Q_{n}} \chi_{B}\left(\{\omega\}_{n-1}\right)\left[m_{n}-m_{n-1}\right]\left(\{\omega\}_{n}\right) \\
& =\int_{\bar{\Omega}_{n}^{-} \times \bar{\Omega}_{n}^{+}} \chi_{B}\left(\{\omega\}_{n-1}^{1}\right) \alpha_{n}^{0}\left(\{\omega\}_{n}^{1} ;\{\omega\}_{n}^{2}\right)\left[m_{n}-m_{n-1}\right]\left(\{\omega\}_{n}^{1}\right) \\
& \times\left[m_{n}-m_{n-1}\right]^{+}\left(\{\omega\}_{n}^{2}\right) \mathrm{d}\left[\bar{P}_{n}^{-} \times \bar{P}_{n}^{+}\right] \\
& +\int_{\bar{\Omega}_{n}^{-} \times \bar{\Omega}_{n}^{+}} \chi_{B}\left(\{\omega\}_{n-1}^{2}\right) \alpha_{n}^{0}\left(\{\omega\}_{n}^{1} ;\{\omega\}_{n}^{2}\right)\left[m_{n}-m_{n-1}\right]\left(\{\omega\}_{n}^{2}\right) \\
& \times\left[m_{n}-m_{n-1}\right]^{-}\left(\{\omega\}_{n}^{1}\right) \mathrm{d}\left[\bar{P}_{n}^{-} \times \bar{P}_{n}^{+}\right] .
\end{aligned}
$$

If to take into account the relations

$$
\begin{gathered}
{\left[m_{n}-m_{n-1}\right]\left(\{\omega\}_{n}\right)=m_{n-1}\left(\{\omega\}_{n-1}\right) a_{n}\left(\{\omega\}_{n-1}\right) \eta_{n}\left(\omega_{n}\right),} \\
{\left[m_{n}-m_{n-1}\right]^{+}\left(\{\omega\}_{n}\right)=m_{n-1}\left(\{\omega\}_{n-1}\right) a_{n}\left(\{\omega\}_{n-1}\right) \eta_{n}^{+}\left(\omega_{n}\right),} \\
{\left[m_{n}-m_{n-1}\right]^{-}\left(\{\omega\}_{n}\right)=m_{n-1}\left(\{\omega\}_{n-1}\right) a_{n}\left(\{\omega\}_{n-1}\right) \eta_{n}^{-}\left(\omega_{n}\right),}
\end{gathered}
$$

and introduce the denotations

$$
\begin{aligned}
\theta_{1}\left(\{\omega\}_{n}^{1} ;\{\omega\}_{n}^{2}\right)= & m_{n-1}\left(\{\omega\}_{n-1}^{1}\right) a_{n}\left(\{\omega\}_{n-1}^{1}\right) \\
& \times m_{n-1}\left(\{\omega\}_{n-1}^{2}\right) a_{n}\left(\{\omega\}_{n-1}^{2}\right) \alpha_{n}^{0}\left(\{\omega\}_{n}^{1} ;\{\omega\}_{n}^{2}\right), \\
\theta_{2}\left(\{\omega\}_{n}^{1} ;\{\omega\}_{n}^{2}\right)= & m_{n-1}\left(\{\omega\}_{n-1}^{1}\right) a_{n}\left(\{\omega\}_{n-1}^{1}\right) \\
& \times m_{n-1}\left(\{\omega\}_{n-1}^{2}\right) a_{n}\left(\{\omega\}_{n-1}^{2}\right) \alpha_{n}^{0}\left(\{\omega\}_{n}^{1} ;\{\omega\}_{n}^{2}\right),
\end{aligned}
$$


we obtain

$$
\begin{aligned}
& E^{Q_{n}} \chi_{B}\left(\{\omega\}_{n-1}\right)\left[m_{n}-m_{n-1}\right]\left(\{\omega\}_{n}\right) \\
& =-\int_{\bar{\Omega}_{n}^{-} \times \Omega_{n}^{+}} \chi_{B}\left(\{\omega\}_{n-1}^{1}\right) \theta_{1}\left(\{\omega\}_{n}^{1} ;\{\omega\}_{n}^{2}\right) \eta_{n}^{-}\left(\omega_{n}^{1}\right) \eta_{n}^{+}\left(\omega_{n}^{2}\right) \mathrm{d}\left[\bar{P}_{n}^{-} \times \bar{P}_{n}^{+}\right] \\
& +\int_{\bar{\Omega}_{n}^{-} \times \Omega_{n}^{+}} \chi_{B}\left(\{\omega\}_{n-1}^{2}\right) \theta_{2}\left(\{\omega\}_{n}^{1} ;\{\omega\}_{n}^{2}\right) \eta_{n}^{-}\left(\omega_{n}^{1}\right) \eta_{n}^{+}\left(\omega_{n}^{2}\right) \mathrm{d}\left[\bar{P}_{n}^{-} \times \bar{P}_{n}^{+}\right] \\
& =\int_{\left\{\eta_{n}\left(\omega_{n}^{1}\right) \leq 00\right\} \times\left\{\eta_{n}\left(\omega_{n}^{2}\right)>0\right\}} \mathrm{d}\left[P_{1}\left(\omega_{n}^{1}\right) \times P_{1}\left(\omega_{n}^{2}\right)\right] \eta_{n}^{-}\left(\omega_{n}^{1}\right) \eta_{n}^{+}\left(\omega_{n}^{2}\right) \\
& \times \int_{\Omega_{n-1} \times \Omega_{n-1}} \chi_{B}\left(\{\omega\}_{n-1}^{1}\right)\left[\theta_{1}\left(\{\omega\}_{n}^{1} ;\{\omega\}_{n}^{2}\right)-\theta_{1}\left(\{\omega\}_{n-1}^{2}, \omega \omega_{n}^{1} ;\{\omega\}_{n-1}^{1}, \omega_{n}^{2}\right)\right] \\
& \times \mathrm{d}\left[P_{n-1}\left(\{\omega\}_{n-1}^{1}\right) \times P_{n-1}\left(\{\omega\}_{n-1}^{2}\right)\right] .
\end{aligned}
$$

It is evident that the expression (31) equals zero for every $B \in \overline{\mathcal{F}}_{n-1}$ if and only if as

$$
\theta_{1}\left(\{\omega\}_{n}^{1} ;\{\omega\}_{n}^{2}\right)-\theta_{1}\left(\{\omega\}_{n-1}^{2}, \omega_{n}^{1} ;\{\omega\}_{n-1}^{1}, \omega_{n}^{2}\right)=0 .
$$

The last equality (32) is valid if the equality

$$
\alpha_{n}^{0}\left(\{\omega\}_{n-1}^{1}, \omega_{n}^{1} ;\{\omega\}_{n-1}^{2}, \omega_{n}^{2}\right)=\alpha_{n}^{0}\left(\{\omega\}_{n-1}^{2}, \omega_{n}^{1} ;\{\omega\}_{n-1}^{1}, \omega_{n}^{2}\right)
$$

is true.

Now if for $\alpha_{n}^{2}\left(\{\omega\}_{n}^{1} ;\{\omega\}_{n}^{2}\right)>0$ satisfying the condition

$$
\int_{\bar{\Omega}_{n}^{-} \times \bar{\Omega}_{n}^{+}} \alpha_{n}^{2}\left(\{\omega\}_{n}^{1} ;\{\omega\}_{n}^{2}\right) \mathrm{d}\left[\bar{P}_{n}^{-} \times \bar{P}_{n}^{+}\right]=1
$$

to put

$$
\alpha_{n}^{0}\left(\{\omega\}_{n}^{1} ;\{\omega\}_{n}^{2}\right)=\frac{\alpha_{n}^{2}\left(\{\omega\}_{n}^{1} ;\{\omega\}_{n}^{2}\right)+\alpha_{n}^{2}\left(T_{n}\left(\{\omega\}_{n}^{1} ;\{\omega\}_{n}^{2}\right)\right)}{\varphi_{n}\left(\{\omega\}_{n}^{1} ;\{\omega\}_{n}^{2}\right)},
$$

then

$$
\begin{aligned}
& Q_{n}(A)=\int_{\bar{\Omega}_{n}^{-} \times \bar{\Omega}_{n}^{+}} \chi_{A}\left(\{\omega\}_{n}^{1}\right) \alpha_{n}^{0}\left(\{\omega\}_{n}^{1} ;\{\omega\}_{n}^{2}\right)\left[m_{n}-m_{n-1}\right]^{+}\left(\{\omega\}_{n}^{2}\right) \mathrm{d}\left[\bar{P}_{n}^{-} \times \bar{P}_{n}^{+}\right] \\
& +\int_{\bar{\Omega}_{n}^{-} \times \bar{\Omega}_{n}^{+}} \chi_{A}\left(\{\omega\}_{n}^{2}\right) \alpha_{n}^{0}\left(\{\omega\}_{n}^{1} ;\{\omega\}_{n}^{2}\right)\left[m_{n}-m_{n-1}\right]\left(\{\omega\}_{n}^{1}\right) \mathrm{d}\left[\bar{P}_{n}^{-} \times \bar{P}_{n}^{+}\right]
\end{aligned}
$$

is a probability measure on the $\sigma$-algebra $\overline{\mathcal{F}}_{n}$.

Taking into account the denotation (26) and the formula (35), we obtain that the measure

$$
\begin{aligned}
& Q_{n}(A)=\int_{\bar{\Omega}_{n}^{-} \times \bar{\Omega}_{n}^{+}} \chi_{A}\left(\{\omega\}_{n}^{1}\right) \alpha_{n}^{1}\left(\{\omega\}_{n}^{1} ;\{\omega\}_{n}^{2}\right) \frac{\left[m_{n}-m_{n-1}\right]^{+}\left(\{\omega\}_{n}^{2}\right)}{\varphi_{n}^{1}\left(\{\omega\}_{n}^{1} ;\{\omega\}_{n}^{2}\right)} \mathrm{d}\left[\bar{P}_{n}^{-} \times \bar{P}_{n}^{+}\right] \\
& +\int_{\bar{\Omega}_{n}^{-} \times \bar{\Omega}_{n}^{+}} \chi_{A}\left(\{\omega\}_{n}^{2}\right) \alpha_{n}^{1}\left(\{\omega\}_{n}^{1} ;\{\omega\}_{n}^{2}\right) \frac{\left[m_{n}-m_{n-1}\right]\left(\{\omega\}_{n}^{1}\right)}{\varphi_{n}^{1}\left(\{\omega\}_{n}^{1} ;\{\omega\}_{n}^{2}\right)} \mathrm{d}\left[\bar{P}_{n}^{-} \times \bar{P}_{n}^{+}\right]
\end{aligned}
$$


is a probability measure on the $\sigma$-algebra $\overline{\mathcal{F}}_{n}$, where

$$
\alpha_{n}^{1}\left(\{\omega\}_{n}^{1} ;\{\omega\}_{n}^{2}\right)=\alpha_{n}^{0}\left(\{\omega\}_{n}^{1} ;\{\omega\}_{n}^{2}\right) \varphi_{n}^{1}\left(\{\omega\}_{n}^{1} ;\{\omega\}_{n}^{2}\right)
$$

satisfy the condition

$$
\int_{\bar{\Omega}_{n}^{-} \times \bar{\Omega}_{n}^{+}} \alpha_{n}^{1}\left(\{\omega\}_{n}^{1} ;\{\omega\}_{n}^{2}\right) \mathrm{d}\left[\bar{P}_{n}^{-} \times \bar{P}_{n}^{+}\right]=1,
$$

due to the condition

$$
\int_{\bar{\Omega}_{n}^{-} \times \bar{\Omega}_{n}^{+}} \alpha_{n}^{2}\left(\{\omega\}_{n}^{1} ;\{\omega\}_{n}^{2}\right) \mathrm{d}\left[\bar{P}_{n}^{-} \times \bar{P}_{n}^{+}\right]=1 .
$$

So, we described the contraction of the martingale measure $Q$ on the $\sigma$-algebra $\mathcal{F}_{n}$ for which $E^{Q_{n}}\left\{m_{n} \mid \mathcal{F}_{n-1}\right\}=m_{n-1}$. It has the representation (37) with the strictly positive random values $\alpha_{n}^{1}\left(\{\omega\}_{n}^{1} ;\{\omega\}_{n}^{2}\right), \alpha_{n}^{2}\left(\{\omega\}_{n}^{1} ;\{\omega\}_{n}^{2}\right)$ satisfying conditions (39), (40).

Since $\Omega_{1}^{0}$ is a separable metric space, then it has an exhaustive decomposition. This is true for $\Omega_{n}$ which is also separable metric space for every $n=\overline{2, N}$. On the probability space $\left\{\bar{\Omega}_{n}^{-} \times \bar{\Omega}_{n}^{+}, \overline{\mathcal{F}}_{n}^{-} \times \overline{\mathcal{F}}_{n}^{+}, \bar{P}_{n}^{-} \times \bar{P}_{n}^{+}\right\}$, for every integrable finite valued random value $f\left(\{\omega\}_{n}^{1} ;\{\omega\}_{n}^{2}\right)$ the sequence $E^{\bar{\mu}_{n}}\left\{f\left(\{\omega\}_{n}^{1} ;\{\omega\}_{n}^{2}\right) \mid \overline{\mathcal{F}}_{m}\right\}$ converges to $f\left(\{\omega\}_{n}^{1} ;\{\omega\}_{n}^{2}\right)$ with probability one, as $m \rightarrow \infty$, since it is a regular martingale. It is evident that for those $B_{m, k s}$ for which $\bar{\mu}_{n}\left(B_{m, k s}\right) \neq 0$, $\bar{\mu}_{n}=\bar{P}_{n}^{-} \times \bar{P}_{n}^{+}$,

$$
E^{\bar{\mu}}\left\{f\left(\{\omega\}_{n}^{1} ;\{\omega\}_{n}^{2}\right) \mid \overline{\mathcal{F}}_{m}\right\}=\frac{\int_{B_{m, k s}} f\left(\{\omega\}_{n}^{1} ;\{\omega\}_{n}^{2}\right) \mathrm{d} \bar{\mu}_{n}}{\bar{\mu}_{n}\left(B_{m, k s}\right)}, \quad\left(\{\omega\}_{n}^{1} ;\{\omega\}_{n}^{2}\right) \in B_{m, k s} .
$$

Denote $D_{0}=\bigcup_{m, k, s, \mu\left(B_{m, k s}\right)=0} B_{m, k s}$. It is evident that $\bar{\mu}_{n}\left(D_{0}\right)=0$. For every $\left(\{\omega\}_{n}^{1} ;\{\omega\}_{n}^{2}\right) \in \bar{\Omega}_{n}^{-} \times \bar{\Omega}_{n}^{+} \backslash D_{0}$, the formula (41) is well defined and is finite. Let $D_{1}$ be the subset of the set $\bar{\Omega}_{n}^{-} \times \bar{\Omega}_{n}^{+} \backslash D_{0}$, where the limit of the left hand side of the formula (41) does not exists. Then, $\bar{\mu}_{n}\left(D_{1}\right)=0$. For every $\left(\{\omega\}_{n}^{1} ;\{\omega\}_{n}^{2}\right) \in \bar{\Omega}_{n}^{-} \times \bar{\Omega}_{n}^{+} \backslash\left(D_{0} \cup D_{1}\right)$, the right hand side of the formula (41) converges to $f\left(\{\omega\}_{n}^{1} ;\{\omega\}_{n}^{2}\right)$. For $\left(\{\omega\}_{n}^{1} ;\{\omega\}_{n}^{2}\right) \in \bar{\Omega}_{n}^{-} \times \bar{\Omega}_{n}^{+} \backslash\left(D_{0} \cup D_{1}\right)$, denote $A_{m}=A_{m}\left(\{\omega\}_{n}^{1} ;\{\omega\}_{n}^{2}\right)$ those set $B_{m, k s}$ for which $\left(\{\omega\}_{n}^{1} ;\{\omega\}_{n}^{2}\right) \in B_{m, k s}$ for a certain $k, s$. Then, for every integrable finite valued $f\left(\{\omega\}_{n}^{1} ;\{\omega\}_{n}^{2}\right)$

$$
\lim _{m \rightarrow \infty} \frac{\int_{A_{m}} f\left(\{\omega\}_{n}^{1} ;\{\omega\}_{n}^{2}\right) \mathrm{d} \bar{\mu}_{n}}{\bar{\mu}_{n}\left(A_{m}\right)}=f\left(\{\omega\}_{n}^{1} ;\{\omega\}_{n}^{2}\right) .
$$

Choose the sequence

$$
\begin{aligned}
& \alpha_{n}^{2, \varepsilon_{m}}\left(\{\omega\}_{n}^{1} ;\{\omega\}_{n}^{2}\right) \\
& =\left(1-\varepsilon_{m}\right) \frac{\chi_{A_{m}}\left(\{\omega\}_{n}^{1} ;\{\omega\}_{n}^{2}\right)}{\bar{\mu}_{n}\left(A_{m}\right)}+\varepsilon_{m} \frac{\chi_{\bar{\Omega}_{n}^{-} \times \bar{\Omega}_{n}^{+} \backslash A_{m}}\left(\{\omega\}_{n}^{1} ;\{\omega\}_{n}^{2}\right)}{\bar{\mu}_{n}\left(\bar{\Omega}_{n}^{-} \times \bar{\Omega}_{n}^{+} \backslash A_{m}\right)},
\end{aligned}
$$

where $0<\varepsilon_{m}<1, \lim _{m \rightarrow \infty} \varepsilon_{m}=0$. Then the sequence $\alpha_{n}^{2, \varepsilon_{m}}\left(\{\omega\}_{n}^{1} ;\{\omega\}_{n}^{2}\right)$ satisfy the 
condition (40). Let us consider the sequence

$$
\begin{aligned}
& \alpha_{n}^{0, \varepsilon_{m}}\left(\{\omega\}_{n}^{1} ;\{\omega\}_{n}^{2}\right) \\
& =\frac{1}{\varphi_{n}\left(\{\omega\}_{n}^{1} ;\{\omega\}_{n}^{2}\right)}\left[\left(1-\varepsilon_{m}\right) \frac{\chi_{A_{m}}\left(\{\omega\}_{n}^{1} ;\{\omega\}_{n}^{2}\right)}{\bar{\mu}_{n}\left(A_{m}\right)}+\varepsilon_{m} \frac{\chi_{\bar{\Omega}_{n}^{-} \times \bar{\Omega}_{n}^{+} \backslash A_{m}}\left(\{\omega\}_{n}^{1} ;\{\omega\}_{n}^{2}\right)}{\bar{\mu}_{n}\left(\bar{\Omega}_{n}^{-} \times \bar{\Omega}_{n}^{+} \backslash A_{m}\right)}\right] \\
& +\frac{1}{\varphi_{n}\left(\{\omega\}_{n}^{1} ;\{\omega\}_{n}^{2}\right)}\left[\left(1-\varepsilon_{m}\right) \frac{\chi_{A_{m}}\left(T_{n}\left(\{\omega\}_{n}^{1} ;\{\omega\}_{n}^{2}\right)\right)}{\bar{\mu}_{n}\left(A_{m}\right)}+\varepsilon_{m} \frac{\chi_{\bar{\Omega}_{n}^{-} \times \bar{\Omega}_{n}^{+} \backslash A_{m}}\left(T_{n}\left(\{\omega\}_{n}^{1} ;\{\omega\}_{n}^{2}\right)\right)}{\bar{\mu}_{n}\left(\bar{\Omega}_{n}^{-} \times \bar{\Omega}_{n}^{+} \backslash A_{m}\right)}\right] .
\end{aligned}
$$

Then the contraction of the sequence of martingale measures $Q^{\varepsilon_{m}}$ generated by sequence (44) on the $\sigma$-algebra $\mathcal{F}_{n}$ is given by the formula

$$
\begin{aligned}
& E^{Q_{n}^{\varepsilon_{m}}} f(\omega)=\int_{\bar{\Omega}_{n}^{-}} \int_{\bar{\Omega}_{n}^{+}} f\left(\{\omega\}_{n}^{1}\right) \alpha_{n}^{0, \varepsilon_{m}}\left(\{\omega\}_{n}^{1} ;\{\omega\}_{n}^{2}\right)\left[m_{n}-m_{n-1}\right]^{+}\left(\{\omega\}_{n}^{2}\right) \mathrm{d} \bar{\mu}_{n} \\
& +\int_{\bar{\Omega}_{n}^{-} \bar{\Omega}_{n}^{+}} f\left(\{\omega\}_{n}^{2}\right) \alpha_{n}^{0, \varepsilon_{m}}\left(\{\omega\}_{n}^{1} ;\{\omega\}_{n}^{2}\right)\left[m_{n}-m_{n-1}\right]^{-}\left(\{\omega\}_{n}^{1}\right) \mathrm{d} \bar{\mu}_{n} \\
& =\left(1-\varepsilon_{m}\right) \frac{\int_{A_{m}} f\left(\{\omega\}_{n}^{1}\right) v_{n}^{1}\left(\{\omega\}_{n}^{1} ;\{\omega\}_{n}^{2}\right) \mathrm{d} \bar{\mu}_{n}}{\bar{\mu}_{n}\left(A_{m}\right)} \\
& +\left(1-\varepsilon_{m}\right) \frac{\int_{A_{m}} f\left(\{\omega\}_{n}^{2}\right) v_{n}^{2}\left(\{\omega\}_{n}^{1} ;\{\omega\}_{n}^{2}\right) \mathrm{d} \bar{\mu}_{n}}{\bar{\mu}_{n}\left(A_{m}\right)} \\
& +\varepsilon_{m} \frac{\int_{\bar{\Omega}_{n}^{-} \times \bar{\Omega}_{n}^{+} \backslash A_{m}} f\left(\{\omega\}_{n}^{1}\right) v_{n}^{1}\left(\{\omega\}_{n}^{1} ;\{\omega\}_{n}^{2}\right) \mathrm{d} \bar{\mu}_{n}}{\bar{\mu}_{n}\left(\bar{\Omega}_{n}^{-} \times \bar{\Omega}_{n}^{+} \backslash A_{m}\right)} \\
& +\varepsilon_{m} \frac{\int_{\Omega_{n}^{-} \times \bar{\Omega}_{n}^{+} \backslash A_{m}} f\left(\{\omega\}_{n}^{2}\right) v_{n}^{2}\left(\{\omega\}_{n}^{1} ;\{\omega\}_{n}^{2}\right) \mathrm{d} \bar{\mu}_{n}}{\bar{\mu}_{n}\left(\bar{\Omega}_{n}^{-} \times \bar{\Omega}_{n}^{+} \backslash A_{m}\right)} \\
& +\left(1-\varepsilon_{m}\right) \frac{\int_{\bar{\Omega}_{n}^{-} \times \bar{\Omega}_{n}^{+}} \chi_{A_{m}}\left(T_{n}\left(\{\omega\}_{n}^{1} ;\{\omega\}_{n}^{2}\right)\right) f\left(\{\omega\}_{n}^{1}\right) v_{n}^{1}\left(\{\omega\}_{n}^{1} ;\{\omega\}_{n}^{2}\right) \mathrm{d} \bar{\mu}_{n}}{\bar{\mu}_{n}\left(A_{m}\right)} \\
& +\left(1-\varepsilon_{m}\right) \frac{\int_{\bar{\Omega}_{n}^{-} \times \bar{\Omega}_{n}^{+}} \chi_{A_{m}}\left(T_{n}\left(\{\omega\}_{n}^{1} ;\{\omega\}_{n}^{2}\right)\right) f\left(\{\omega\}_{n}^{2}\right) v_{n}^{2}\left(\{\omega\}_{n}^{1} ;\{\omega\}_{n}^{2}\right) \mathrm{d} \bar{\mu}_{n}}{\bar{\mu}_{n}\left(A_{m}\right)} \\
& +\varepsilon_{m} \frac{\int_{\bar{\Omega}_{n}^{-} \times \bar{\Omega}_{n}^{+}} \chi_{\bar{\Omega}_{n}^{-} \times \bar{\Omega}_{n}^{+} \backslash A_{m}}\left(T_{n}\left(\{\omega\}_{n}^{1} ;\{\omega\}_{n}^{2}\right)\right) f\left(\{\omega\}_{n}^{1}\right) v_{n}^{1}\left(\{\omega\}_{n}^{1} ;\{\omega\}_{n}^{2}\right) \mathrm{d} \bar{\mu}_{n}}{\bar{\mu}_{n}\left(\bar{\Omega}_{n}^{-} \times \bar{\Omega}_{n}^{+} \backslash A_{m}\right)} \\
& +\varepsilon_{m} \frac{\int_{\bar{\Omega}_{n}^{-} \times \bar{\Omega}_{n}^{+}} \chi_{\bar{\Omega}_{n}^{-} \times \bar{\Omega}_{n}^{+} \backslash A_{m}}\left(T_{n}\left(\{\omega\}_{n}^{1} ;\{\omega\}_{n}^{2}\right)\right) f\left(\{\omega\}_{n}^{2}\right) v_{n}^{2}\left(\{\omega\}_{n}^{1} ;\{\omega\}_{n}^{2}\right) \mathrm{d} \bar{\mu}_{n}}{\bar{\mu}_{n}\left(\bar{\Omega}_{n}^{-} \times \bar{\Omega}_{n}^{+} \backslash A_{m}\right)} .
\end{aligned}
$$

Due to the invariance of the measure $\bar{\mu}_{n}$ relative to the transformation $T_{n}$ we have 


$$
\begin{aligned}
& \left(1-\varepsilon_{m}\right) \frac{\int_{\bar{\Omega}_{n}^{-} \times \bar{\Omega}_{n}^{+}} \chi_{A_{m}}\left(T_{n}\left(\{\omega\}_{n}^{1} ;\{\omega\}_{n}^{2}\right)\right) f\left(\{\omega\}_{n}^{1}\right) v_{n}^{1}\left(\{\omega\}_{n}^{1} ;\{\omega\}_{n}^{2}\right) \mathrm{d} \bar{\mu}_{n}}{\bar{\mu}_{n}\left(A_{m}\right)} \\
& +\left(1-\varepsilon_{m}\right) \frac{\int_{\bar{\Omega}_{\times}^{-} \times \bar{\Omega}_{n}^{+}} \chi_{A_{m}}\left(T_{n}\left(\{\omega\}_{n}^{1} ;\{\omega\}_{n}^{2}\right)\right) f\left(\{\omega\}_{n}^{2}\right) v_{n}^{2}\left(\{\omega\}_{n}^{1} ;\{\omega\}_{n}^{2}\right) \mathrm{d} \bar{\mu}_{n}}{\bar{\mu}_{n}\left(A_{m}\right)} \\
& =\left(1-\varepsilon_{m}\right) \frac{\int_{A_{m}} f\left(T_{n}^{1}\left(\{\omega\}_{n}^{1}\right)\right) v_{n}^{1}\left(T_{n}\left(\{\omega\}_{n}^{1} ;\{\omega\}_{n}^{2}\right)\right) \mathrm{d} \bar{\mu}_{n}}{\bar{\mu}_{n}\left(A_{m}\right)} \\
& +\left(1-\varepsilon_{m}\right) \frac{\int_{A_{m}} f\left(T_{n}^{2}\left(\{\omega\}_{n}^{2}\right)\right) v_{n}^{2}\left(T_{n}\left(\{\omega\}_{n}^{1} ;\{\omega\}_{n}^{2}\right)\right) \mathrm{d} \bar{\mu}_{n}}{\bar{\mu}_{n}\left(A_{m}\right)} .
\end{aligned}
$$

From the equalities (45), (46) it follows that

$$
\begin{aligned}
& \lim _{m \rightarrow \infty} E^{Q_{n}^{\varepsilon_{m}}} f_{n}\left(\{\omega\}_{n}\right) \\
& =f\left(\{\omega\}_{n}^{1}\right) v_{n}^{1}\left(\{\omega\}_{n}^{1} ;\{\omega\}_{n}^{2}\right)+f\left(\{\omega\}_{n}^{2}\right) v_{n}^{2}\left(\{\omega\}_{n}^{1} ;\{\omega\}_{n}^{2}\right) \\
& +f\left(T_{n}^{1}\left(\{\omega\}_{n}^{1}\right)\right) v_{n}^{1}\left(T_{n}\left(\{\omega\}_{n}^{1} ;\{\omega\}_{n}^{2}\right)\right) \\
& +f\left(T_{n}^{2}\left(\{\omega\}_{n}^{2}\right)\right) v_{n}^{2}\left(T_{n}\left(\{\omega\}_{n}^{1} ;\{\omega\}_{n}^{2}\right)\right), n=\overline{1, N} .
\end{aligned}
$$

Theorem 2 is proved.

Theorem 3. On the probability space $\{\Omega, \mathcal{F}, P\}$ with the filtration $\mathcal{F}_{n}$ on it, let $\Omega_{1}^{0}$ be a complete separable metric space. Suppose that $f_{n}\left(\{\omega\}_{n}\right)$ is a nonnegative integrable $\mathcal{F}_{n}$-measurable random value, satisfying the condition $E^{Q^{n}} f_{n}\left(\{\omega\}_{n}\right) \leq 1, Q^{n} \in M_{n}$. Then, there exists a $\mathcal{F}_{n-1}$-measurable random value $\alpha_{n}$, depending on $f_{n}\left(\{\omega\}_{n}\right)$, such that

$$
f_{n}\left(\{\omega\}_{n}\right) \leq 1+\alpha_{n}\left(\{\omega\}_{n-1}\right)\left[m_{n}-m_{n-1}\right]\left(\{\omega\}_{n}\right), \quad\{\omega\}_{n} \in \Omega_{n} .
$$

Proof. First, let us consider the case $n=1$. From Theorem 2, we have the inequality

$$
\begin{gathered}
f_{1}\left(\omega_{1}\right) \frac{\left[m_{1}-1\right]^{+}\left(\omega_{2}\right)}{\left[m_{1}-1\right]^{-}\left(\omega_{1}\right)+\left[m_{1}-1\right]^{+}\left(\omega_{2}\right)} \\
+f_{1}\left(\omega_{2}\right) \frac{\left[m_{1}-1\right]^{-}\left(\omega_{1}\right)}{\left[m_{1}-1\right]^{-}\left(\omega_{1}\right)+\left[m_{1}-1\right]^{+}\left(\omega_{2}\right)} \leq 1, \\
\left(\omega_{1}, \omega_{2}\right) \in \Omega_{1}^{-} \times \Omega_{1}^{+},
\end{gathered}
$$

where $\Omega_{1}^{-}=\left\{\omega_{1} \in \Omega_{1}^{0},\left[m_{1}-1\right]\left(\omega_{1}\right) \leq 0\right\}, \Omega_{1}^{+}=\left\{\omega_{2} \in \Omega_{1}^{0},\left[m_{1}-1\right]\left(\omega_{2}\right)>0\right\}$.

Let us denote $\eta_{1}(\omega)=\left[m_{1}-1\right](\omega)$. Then, the formula (49) is written in the form

$$
f_{1}\left(\omega_{1}\right) \frac{\eta_{1}^{+}\left(\omega_{2}\right)}{\eta_{1}^{-}\left(\omega_{1}\right)+\eta_{1}^{+}\left(\omega_{2}\right)}+\frac{\eta_{1}^{-}\left(\omega_{1}\right)}{\eta_{1}^{-}\left(\omega_{1}\right)+\eta_{1}^{+}\left(\omega_{2}\right)} f_{1}\left(\omega_{2}\right) \leq 1, \omega_{1} \in \Omega_{1}^{-}, \omega_{2} \in \Omega_{1}^{+} .
$$

From the inequalities (50), we obtain the inequalities 


$$
\begin{gathered}
f_{1}\left(\omega_{2}\right) \leq 1+\frac{1-f_{1}\left(\omega_{1}\right)}{\eta_{1}^{-}\left(\omega_{1}\right)} \eta_{1}^{+}\left(\omega_{2}\right), \\
\eta_{1}^{-}\left(\omega_{1}\right)>0, \quad \eta_{1}^{+}\left(\omega_{2}\right)>0, \quad \omega_{1} \in \Omega_{1}^{-}, \quad \omega_{2} \in \Omega_{1}^{+} .
\end{gathered}
$$

Two cases are possible: a) for all $\omega_{1} \in \Omega_{n}^{-}, \quad f_{1}\left(\omega_{1}\right) \leq 1$; b) there exists $\omega_{1} \in \Omega_{1}^{-}$ such that $f_{1}\left(\omega_{1}\right)>1$. First, let us consider the case a).

Since the inequalities (51) are valid for every value $\frac{1-f_{1}\left(\omega_{1}\right)}{\eta_{1}^{-}\left(\omega_{1}\right)}$, as $\eta_{1}^{-}\left(\omega_{1}\right)>0$, and $f_{1}\left(\omega_{1}\right) \leq 1, \omega_{1} \in \Omega_{1}^{-}$, then, if to denote

$$
\alpha_{1}=\inf _{\left\{\omega_{1}, \eta_{1}^{-}\left(\omega_{1}\right)>0\right\}} \frac{1-f_{1}\left(\omega_{1}\right)}{\eta_{1}^{-}\left(\omega_{1}\right)},
$$

we have $0 \leq \alpha_{1}<\infty$ and

$$
f_{1}\left(\omega_{2}\right) \leq 1+\alpha_{1} \eta_{1}^{+}\left(\omega_{2}\right), \quad \eta_{1}^{+}\left(\omega_{2}\right)>0, \quad \omega_{2} \in \Omega_{1}^{+} .
$$

From the definition of $\alpha_{1}$, we obtain the inequalities

$$
f_{1}\left(\omega_{1}\right) \leq 1-\alpha_{1} \eta_{1}^{-}\left(\omega_{1}\right), \quad \eta_{1}^{-}\left(\omega_{1}\right)>0, \quad \omega_{1} \in \Omega_{1}^{-} .
$$

Now, if $\eta_{1}^{-}\left(\omega_{1}\right)=0$ for some $\omega_{1} \in \Omega_{1}^{-}$, then in this case $f_{1}\left(\omega_{1}\right) \leq 1$. All these inequalities give the inequalities

$$
f_{1}(\omega) \leq 1+\alpha_{1} \eta_{1}(\omega), \omega \in \Omega_{1}^{-} \cup \Omega_{1}^{+} .
$$

Consider the case b). From the inequality (51), we obtain the inequalities

$$
\begin{gathered}
f_{1}\left(\omega_{2}\right) \leq 1-\frac{1-f_{1}\left(\omega_{1}\right)}{-\eta_{1}^{-}\left(\omega_{1}\right)} \eta_{1}^{+}\left(\omega_{2}\right), \\
\eta_{1}^{-}\left(\omega_{1}\right)>0, \quad \eta_{1}^{+}\left(\omega_{2}\right)>0, \quad \omega_{1} \in \Omega_{1}^{-}, \quad \omega_{2} \in \Omega_{1}^{+} .
\end{gathered}
$$

The inequalities (57) give the inequalities

$$
\frac{1-f_{1}\left(\omega_{1}\right)}{-\eta_{1}^{-}\left(\omega_{1}\right)} \leq \inf _{\left\{\omega_{2}, \eta_{1}^{+}\left(\omega_{2}\right)>0\right\}} \frac{1}{\eta_{1}^{+}\left(\omega_{2}\right)}<\infty, \quad \eta_{1}^{-}\left(\omega_{1}\right)>0, \quad \omega_{1} \in \Omega_{1}^{-} .
$$

Let us define $\alpha_{1}=\sup _{\left\{\omega_{1}, \eta_{1}^{-}\left(\omega_{1}\right)>0\right\}} \frac{1-f_{1}\left(\omega_{1}\right)}{-\eta_{1}^{-}\left(\omega_{1}\right)}<\infty$. Then, from (57) we obtain the inequalities

$$
f_{1}\left(\omega_{2}\right) \leq 1-\alpha_{1} \eta_{1}^{+}\left(\omega_{2}\right), \quad \eta_{1}^{+}\left(\omega_{2}\right)>0, \quad \omega_{2} \in \Omega_{1}^{+} .
$$

From the definition of $\alpha_{1}$, we have the inequalities

$$
f_{1}\left(\omega_{1}\right) \leq 1+\alpha_{1} \eta_{1}^{-}\left(\omega_{1}\right), \quad \eta_{1}^{-}\left(\omega_{1}\right)>0, \quad \omega_{1} \in \Omega_{1}^{-} .
$$

The inequalities (60), (61) give the inequalities

$$
f_{1}(\omega) \leq 1-\alpha_{1} \eta_{1}(\omega), \quad \omega \in \Omega_{1}^{-} \cup \Omega_{1}^{+} .
$$

Theorem 3 in the case $n=1$ is proved, since the set $\Omega_{1}^{-} \cup \Omega_{1}^{+}$has the probability one.

Now let us consider the case of arbitrary $2 \leq n \leq N$. In this case we have the inequality 


$$
\begin{aligned}
& f\left(\{\omega\}_{n}^{1}\right) v_{n}^{1}\left(\{\omega\}_{n}^{1} ;\{\omega\}_{n}^{2}\right)+f\left(\{\omega\}_{n}^{2}\right) v_{n}^{2}\left(\{\omega\}_{n}^{1} ;\{\omega\}_{n}^{2}\right) \\
& +f\left(T_{n}^{1}\left(\{\omega\}_{n}^{1}\right)\right) v_{n}^{1}\left(T_{n}\left(\{\omega\}_{n}^{1} ;\{\omega\}_{n}^{2}\right)\right) \\
& +f\left(T_{n}^{2}\left(\{\omega\}_{n}^{2}\right)\right) v_{n}^{2}\left(T_{n}\left(\{\omega\}_{n}^{1} ;\{\omega\}_{n}^{2}\right)\right) \leq 1 .
\end{aligned}
$$

Let us put in this inequality $\{\omega\}_{n-1}^{1}=\{\omega\}_{n-1}^{2}=\{\omega\}_{n-1}$, then the inequality (63) is transformed into the inequality

$$
\begin{aligned}
& f_{n}\left(\{\omega\}_{n-1}, \omega_{n}^{1}\right) \frac{\eta_{n}^{+}\left(\omega_{n}^{2}\right)}{\eta_{n}^{-}\left(\omega_{n}^{1}\right)+\eta_{n}^{+}\left(\omega_{n}^{2}\right)}+\frac{\eta_{n}^{-}\left(\omega_{n}^{1}\right)}{\eta_{n}^{-}\left(\omega_{n}^{1}\right)+\eta_{n}^{+}\left(\omega_{n}^{2}\right)} f_{n}\left(\{\omega\}_{n-1}, \omega_{n}^{2}\right) \leq 1, \\
& \left(\{\omega\}_{n-1}, \omega_{n}^{1}\right) \in \Omega_{n}^{-}, \quad\left(\{\omega\}_{n-1}, \omega_{n}^{2}\right) \in \Omega_{n}^{+} .
\end{aligned}
$$

Taking into account the first part of the proof of Theorem 3 from the inequality (64) we obtain

$$
f_{n}\left(\{\omega\}_{n}\right) \leq 1+\alpha_{1} \eta_{n}\left(\omega_{n}\right)=1+\frac{\alpha_{1}\left[m_{n}-m_{n-1}\right]\left(\{\omega\}_{n}\right)}{m_{n-1}\left(\{\omega\}_{n-1}\right) a_{n}\left(\{\omega\}_{n-1}\right)},
$$

where the constant $\alpha_{1}$ is the same as in the first part of the proof of Theorem 3. Theorem 3 is completely proved.

Theorem 4. On the probability space $\{\Omega, \mathcal{F}, P\}$ with the filtration $\mathcal{F}_{n}$ on it, let $\Omega_{1}^{0}$ be a complete separable metric space. Then, every nonnegative supermartingale $\left\{f_{n}, \mathcal{F}_{n}\right\}_{n=0}^{N}$ is a local regular one, that is, the optional decomposition for it is valid.

Proof. Without loss of generality, we assume that $f_{n} \geq d_{0}>0$. From the last fact, we obtain

$$
E^{Q^{n}} \frac{f_{n}}{f_{n-1}} \leq 1, \quad Q^{n} \in M_{n}, \quad n=\overline{1, \infty} .
$$

The inequalities (66) and Theorems 3, 4 [1], [17] prove Theorem 4.

Theorem 5. On the probability space $\{\Omega, \mathcal{F}, P\}$ with the filtration $\mathcal{F}_{n}$ on it, let $\Omega_{1}^{0}$ be a complete separable metric space. Then, every bounded from below super-martingale $\left\{f_{n}, \mathcal{F}_{n}\right\}_{n=0}^{\infty}$ is a local regular one.

Proof. Since the super-martingale $\left\{f_{n}, \mathcal{F}_{n}\right\}_{n=0}^{N}$ is bounded from below, then there exists a real number $C_{0}$ such that $f_{n}+C_{0}>0$. If to consider the super-martingale $\left\{f_{n}+C_{0}, \mathcal{F}_{n}\right\}_{n=0}^{\infty}$, then all conditions of Theorem 4 are true. Theorem 5 is proved.

Theorem 6. On the probability space $\{\Omega, \mathcal{F}, P\}$ with the filtration $\mathcal{F}_{n}$ on it, let $\Omega_{1}^{0}$ be a complete separable metric space. Suppose that the evolution of the risk asset is defined by the formula (2) and the non risk asset evolve by the law $B_{n}=1, n=\overline{0, N}$. If the nonnegative payoff function $f_{N}$ is $\mathcal{F}_{N}$ measurable integrable random value relative to every martingale measure and satisfying the conditions Theorem 16 from [17], then the fair price of super-hedge is given by the formula

$$
\sup _{Q \in M} \int_{\Omega} f_{N} \mathrm{~d} Q=\sup _{v \in \mathcal{V}} \int_{\Omega} f_{N} \mathrm{~d} v_{v}
$$




\section{Interval of Non-Arbitrage Prices for a Wide Class of Evolutions of Risky Assets}

In the papers [2], [3] the range of non arbitrage prices are established. In the paper [2], for the Levy exponential model, the price of super-hedge for call option coincides with the price of the underlying asset under the assumption that the Levy process has unlimited variation, does not contain a Brownian component, with negative jumps of arbitrary magnitude. The same result is true obtained in the paper [3] if the process describing the evolution of the underlying asset is a diffusion process with the jumps described by Poisson jump process. In these papers the evolution is described by continuous processes. Below we consider the discrete evolution of risky assets that is more realistic from the practical point of view.

Theorem 7. On the probability space $\left\{\Omega_{1}^{0}, \mathcal{F}_{1}^{0}, P_{1}^{0}\right\}$, where $\Omega_{1}^{0}$ is a separable metric space, $\mathcal{F}_{1}^{0}$ is a Borel $\sigma$-algebra on $\Omega_{1}^{0}, P_{1}^{0}$ is a probability measure on $\mathcal{F}_{1}^{0}$, let the random values $\xi_{i}(\omega) \geq 0, \quad i=\overline{1, N}$, satisfies the conditions.

1) $0<P_{1}^{0}\left(0 \leq \xi_{i}\left(\omega_{1}\right) \leq 1\right)<1$, there exists $\omega_{1}$ and $\omega_{2}$ such that $\xi_{i}\left(\omega_{1}\right)=0$, $\xi_{i}\left(\omega_{2}\right)=1, \quad i=\overline{1, N}$;

2) for every $1<t<\infty, \quad P_{1}^{0}\left(\xi_{i}\left(\omega_{1}\right)>t\right)>0, \quad i=\overline{1, N}$.

Suppose that the evolution of risk asset is given by the formula (2) with $a_{1}=1$, where $\eta_{i}\left(\omega_{i}\right)=\xi_{i}\left(\omega_{i}\right)-1$, and on the probability space $\left\{\Omega_{i}^{0}, \mathcal{F}_{i}^{0}, P_{i}^{0}\right\}$, the random value $\xi_{i}\left(\omega_{i}\right)$ has the same distribution law as the random value $\xi_{i}\left(\omega_{1}\right)$, $i=\overline{1, N}$, on the probability space $\left\{\Omega_{1}^{0}, \mathcal{F}_{1}^{0}, P_{1}^{0}\right\}$. If the nonnegative payoff function $f(x), x \in[0, \infty)$, satisfies the conditions:

1) $f(0)=0, f(x) \leq a x, \lim _{x \rightarrow \infty} \frac{f(x)}{x}=a, a>0$, then

$$
\sup _{P \in M} E^{P} f\left(S_{N}\right)=a S_{0} .
$$

If, in addition, the nonnegative payoff function $f(x)$ is a convex down one, then

$$
\inf _{P \in M} E^{P} f\left(S_{N}\right)=f\left(S_{0}\right),
$$

where $M$ is the set of equivalent martingale measures for the evolution of risk asset $S_{n}, n=\overline{1, N}$. The interval of non-arbitrage prices of contingent liability $f\left(S_{N}\right)$ coincides with the set $\left[f\left(S_{0}\right), a S_{0}\right]$.

Proof. Due to Theorem 1 and 6 we have

$$
\sup _{Q \in M} \int_{\Omega} f\left(S_{N}\right) \mathrm{d} Q=\sup _{v \in \mathcal{V}} \int_{\Omega} f\left(S_{N}\right) \mathrm{d} v_{v},
$$

where

$$
\begin{aligned}
& \sup _{v \in \mathcal{V}} \int_{\Omega} f\left(S_{N}\right) \mathrm{d} v_{v} \\
& =\sup _{v \in \mathcal{V}} \sum_{i_{1}=1, \cdots, i_{N}=1}^{2} f\left(S_{0} \prod_{s=1}^{N}\left(1+a_{s}\left(\omega_{1}^{i_{1}}, \cdots, \omega_{s-1}^{i_{s-1}}\right) \eta_{s}\left(\omega_{s}^{i_{s}}\right)\right)\right) \prod_{s=1}^{N} \frac{\left|\xi_{s}\left(\omega_{s}^{i_{s}+1}\right)-1\right|}{\xi_{s}\left(\omega_{s}^{2}\right)-\xi_{s}\left(\omega_{s}^{1}\right)},
\end{aligned}
$$

and we used the denotations $\left(\xi_{s}\left(\omega_{s}^{1}\right)-1\right)^{-}=1-\xi_{s}\left(\omega_{s}^{1}\right)$, 
$\left(\xi_{s}\left(\omega_{s}^{2}\right)-1\right)^{+}=\xi_{s}\left(\omega_{s}^{2}\right)-1, \quad \omega_{s}^{1} \in \Omega_{s}^{-}, \omega_{s}^{2} \in \Omega_{s}^{+}, \omega_{s}^{3}=\omega_{s}^{1}$. From the inequality $f\left(S_{N}\right) \leq a S_{N}$ we have

$$
\sup _{Q \in M} \int_{\Omega} f\left(S_{N}\right) \mathrm{d} Q \leq a S_{0} .
$$

To prove the inverse inequality we use the inequality

$$
\begin{aligned}
& \sup _{Q \in M} \int_{\Omega} f\left(S_{N}\right) \mathrm{d} Q \\
& \geq f\left(S_{0} \xi_{1}\left(\omega_{1}^{1}\right)\right) \frac{\xi_{1}\left(\omega_{1}^{2}\right)-1}{\xi_{1}\left(\omega_{1}^{2}\right)-\xi_{1}\left(\omega_{1}^{1}\right)}+f\left(S_{0} \xi_{1}\left(\omega_{1}^{2}\right)\right) \frac{1-\xi_{1}\left(\omega_{1}^{1}\right)}{\xi_{1}\left(\omega_{1}^{2}\right)-\xi_{1}\left(\omega_{1}^{1}\right)} .
\end{aligned}
$$

Therefore, putting in the inequality (73) $\xi_{1}\left(\omega_{1}^{1}\right)=0$ we obtain

$$
\sup _{Q \in M} \int_{\Omega} f\left(S_{N}\right) \mathrm{d} Q \geq \lim _{\xi_{1}\left(\omega_{1}^{2}\right) \rightarrow \infty} f\left(S_{0} \xi_{1}\left(\omega_{1}^{2}\right)\right) \frac{1}{\xi_{1}\left(\omega_{1}^{2}\right)}=a S_{0} .
$$

Let us prove the equality (69). Using Jensen inequality [18] we obtain

$$
\inf _{P \in M} E^{P} f\left(S_{N}\right) \geq f\left(E^{P} S_{N}\right)=f\left(S_{0}\right)
$$

Let us prove the inverse inequality

$$
f\left(S_{0} \xi_{1}\left(\omega_{1}^{1}\right)\right) \frac{\xi_{1}\left(\omega_{1}^{2}\right)-1}{\xi_{1}\left(\omega_{1}^{2}\right)-\xi_{1}\left(\omega_{1}^{1}\right)}+f\left(S_{0} \xi_{1}\left(\omega_{1}^{2}\right)\right) \frac{1-\xi_{1}\left(\omega_{1}^{1}\right)}{\xi_{1}\left(\omega_{1}^{2}\right)-\xi_{1}\left(\omega_{1}^{1}\right)} \geq \inf _{P \in M} E^{P} f\left(S_{N}\right) .
$$

Putting in this inequality $\xi_{1}\left(\omega_{1}^{1}\right)=1$ we obtain the needed. The last statement about the interval of non-arbitrage prices follows from [11] and [12]. Theorem 7 is proved.

Theorem 8. On the probability space $\left\{\Omega_{1}^{0}, \mathcal{F}_{1}^{0}, P_{1}^{0}\right\}$, where $\Omega_{1}^{0}$ is a separable metric space, $\mathcal{F}_{1}^{0}$ is a Borel $\sigma$-algebra on $\Omega_{1}^{0}, P_{1}^{0}$ is a probability measure on $\mathcal{F}_{1}^{0}$, let the random values $\xi_{i}\left(\omega_{1}\right) \geq 0, i=\overline{1, N}$, satisfies the conditions.

1) $0<P_{1}^{0}\left(0 \leq \xi_{i}\left(\omega_{1}\right) \leq 1\right)<1$, there exists $\omega_{1}$ and $\omega_{2}$ such that $\xi_{i}\left(\omega_{1}\right)=0$, $\xi_{i}\left(\omega_{2}\right)=1, \quad i=\overline{1, N}$;

2) for every $1<t<\infty, P_{1}^{0}\left(\xi_{i}\left(\omega_{1}\right)>t\right)>0$.

Suppose that the evolution of risk asset is given by the formula (2) with $a_{1}=1$, where $\eta_{i}\left(\omega_{i}\right)=\xi_{i}\left(\omega_{i}\right)-1$, and on the probability space $\left\{\Omega_{i}^{0}, \mathcal{F}_{i}^{0}, P_{i}^{0}\right\}$, the random value $\xi_{i}\left(\omega_{i}\right)$ has the same distribution law as the random value $\xi_{i}\left(\omega_{1}\right)$, $i=\overline{1, N}$, on the probability space $\left\{\Omega_{1}^{0}, \mathcal{F}_{1}^{0}, P_{1}^{0}\right\}$. If the nonnegative payoff function $f(x), x \in[0, \infty)$, satisfies the conditions:

1) $f(0)=K, \quad f(x) \leq K$, then

$$
\sup _{P \in M} \int_{\Omega} E^{P} f\left(S_{N}\right)=K .
$$

If, in addition, the nonnegative payoff function $f(x)$ is a convex down one, then

$$
\inf _{P \in M} E^{P} f\left(S_{N}\right)=f\left(S_{0}\right),
$$

where $M$ is the set of equivalent maqtingale measures for the evolution of risk asset $S_{n}, n=\overline{0, N}$. The interval of non-arbitrage prices of contingent liability 
$f\left(S_{N}\right)$ coincides with the set $\left[f\left(S_{0}\right), K\right]$.

Proof. It is evident that

$$
\sup _{P \in M} E^{P} f\left(S_{N}\right) \leq K .
$$

Since

$$
\begin{aligned}
& \sup _{v \in \mathcal{V}} \int_{\Omega} f\left(S_{N}\right) \mathrm{d} v_{v} \\
& =\sup _{v \in \mathcal{V}} \sum_{i_{1}=1, \cdots, i_{N}=1}^{2} f\left(S_{0} \prod_{s=1}^{N}\left(1+a_{s}\left(\omega_{1}^{i_{1}}, \cdots, \omega_{s-1}^{i_{s-1}}\right) \eta_{s}\left(\omega_{s}^{i_{s}}\right)\right)\right) \prod_{s=1}^{N} \frac{\left|\xi_{s}\left(\omega_{s}^{i_{s}+1}\right)-1\right|}{\xi_{s}\left(\omega_{s}^{2}\right)-\xi_{s}\left(\omega_{s}^{1}\right)},
\end{aligned}
$$

we have

$$
\begin{aligned}
& \sup _{Q \in M} \int_{\Omega} f\left(S_{N}\right) \mathrm{d} Q \\
& \geq f\left(S_{0} \xi_{1}\left(\omega_{1}^{1}\right)\right) \frac{\xi_{1}\left(\omega_{1}^{2}\right)-1}{\xi_{1}\left(\omega_{1}^{2}\right)-\xi_{1}\left(\omega_{1}^{1}\right)}+f\left(S_{0} \xi_{1}\left(\omega_{1}^{2}\right)\right) \frac{1-\xi_{1}\left(\omega_{1}^{1}\right)}{\xi_{1}\left(\omega_{1}^{2}\right)-\xi_{1}\left(\omega_{1}^{1}\right)} .
\end{aligned}
$$

Therefore, putting in the inequality (81) $\xi_{1}\left(\omega_{1}^{1}\right)=0$ we obtain

$$
\begin{aligned}
& \sup _{Q \in M} \int_{\Omega} f\left(S_{N}\right) \mathrm{d} Q \\
& \geq \lim _{\xi_{1}\left(\omega_{1}^{2}\right) \rightarrow \infty}\left[f(0) \frac{\xi_{1}\left(\omega_{1}^{2}\right)-1}{\xi_{1}\left(\omega_{1}^{2}\right)}+f\left(S_{0} \xi_{1}\left(\omega_{1}^{2}\right)\right) \frac{1}{\xi_{1}\left(\omega_{1}^{2}\right)}\right]=f(0)=K .
\end{aligned}
$$

Let us prove the equality (78). Due to convexity of payoff function $f(x)$, using Jensen inequality we obtain

$$
\inf _{P \in M} E^{P} f\left(S_{N}\right) \geq f\left(E^{P} S_{N}\right)=f\left(S_{0}\right) .
$$

Let us prove the inverse inequality

$$
\begin{aligned}
& f\left(S_{0} \xi_{1}\left(\omega_{1}^{1}\right)\right) \frac{\xi_{1}\left(\omega_{1}^{2}\right)-1}{\xi_{1}\left(\omega_{1}^{2}\right)-\xi_{1}\left(\omega_{1}^{1}\right)}+f\left(S_{0} \xi_{1}\left(\omega_{1}^{2}\right)\right) \frac{1-\xi_{1}\left(\omega_{1}^{1}\right)}{\xi_{1}\left(\omega_{1}^{2}\right)-\xi_{1}\left(\omega_{1}^{1}\right)} \\
& \geq \inf _{P \in M} E^{P} f\left(S_{N}\right) .
\end{aligned}
$$

Putting in this inequality $\xi_{1}\left(\omega_{1}^{1}\right)=1$ we obtain the needed. Theorem 8 is proved.

Remark 3. The results obtained in Theorems 7,8 are true if for some $s>1$, $a_{s}\left(\omega_{1}, \cdots, \omega_{s-1}\right)=1$.

Let us give an example of application of the results obtained. Denote $w(t), t \in[0, T]$ standard Brownian motion on the time interval $[0, T]$ with $w(0)=0$. Due to the continuity of $w(t)$ the Winer measure $P$ is concentrated on the Banach space $C([0, T])$ with the norm $\|f\|=\sup _{t \in[0, T]}|f(t)|$,

$f(t) \in C([0, T])$. The space $C([0, T])$ is a complete separable metric space in the metric generated by the introduced norm. Suppose that $0=t_{0}<t_{1}<t_{2}<\cdots<t_{N}=T$. On the probability space $\left\{\Omega_{1}^{0}, \mathcal{F}_{1}^{0}, P_{1}^{0}\right\}$, where $\Omega_{1}^{0}=C([0, T]), \mathcal{F}_{1}^{0}$ is a Borel $\sigma$-algebra on $\Omega_{1}^{0}, P_{1}^{0}=P$ is a probability meas- 
ure on $\mathcal{F}_{1}^{0}$, let us consider the random values $\xi_{i}\left(\omega_{1}\right) \geq 0, i=\overline{1, N}$, where $\xi_{i}\left(\omega_{1}\right)=\mathrm{e}^{\left(\mu-\frac{\sigma^{2}}{2}-r\right)\left(t_{i}-t_{i-1}\right)+\sigma\left(w\left(t_{i}\right)-w\left(t_{i-1}\right)\right)}, i=\overline{1, N}$. The random values $\xi_{i}\left(\omega_{1}\right)$, $i=\overline{1, N}$, are independent between themselves.

The random values $\xi_{i}\left(\omega_{1}\right), i=\overline{1, N}$, generate the evolution of risk asset given by the formula $S_{n}=S_{0} \prod_{i=1}^{n} \xi_{i}\left(\omega_{1}\right)=S_{0} \mathrm{e}^{\left(\mu-\frac{\sigma^{2}}{2}-r\right) t_{n}+\sigma w\left(t_{n}\right)}, n=\overline{1, N}$. Such evolution satisfies the conditions of the Theorems 7, 8.

\section{Models of Evolution of Risky Assets Based on the Discrete Geometric Brownian Motion}

Suppose that the set $t_{0}=0, t_{1}, t_{2}, \cdots, t_{N}=T$ belongs to $[0, \infty)$ with $\Delta t=t_{i}-t_{i-1}>0$ not depending on the index $i$.

On the probability space $\left\{\Omega_{1}^{0}, \mathcal{F}_{1}^{0}, P_{1}^{0}\right\}$, considered in the previous example, let us consider the sequence of random values

$$
\xi_{i}\left(\omega_{1}\right)=\mathrm{e}^{\left(\mu-\frac{\sigma^{2}}{2}-r\right) \Delta t+\sigma\left(w\left(t_{i}\right)-w\left(t_{i-1}\right)\right)}, i=\overline{1, N}
$$

where $w\left(t_{i}\right)$ is a standard Brownian motion with $w(0)=0$,

$E w\left(t_{i}\right) w\left(t_{j}\right)=\min \left\{t_{i}, t_{j}\right\}$. With every sequence real numbers $a_{1}, a_{2}, \cdots, a_{n}, \cdots$, $0 \leq a_{i} \leq 1, \quad i=1, \infty$, let us connect the random process

$$
S_{n}=S_{0} \prod_{i=1}^{n}\left[1+a_{i}\left(\xi_{i}\left(\omega_{1}\right)-1\right)\right], \quad n=\overline{1, N}, \quad S_{0}>0 .
$$

Below we construct the probability space $\{\Omega, \mathcal{F}, P\}$ and the random process $S_{n}, n=\overline{1, N}$, on it, which is equivalent one in the wide sense to the process (86). For this purpose we could do it using the method, presented in section 2. But for further applications, it is more convenient to construct the simple probability space $\{\Omega, \mathcal{F}, P\}$ and the random process on it, which is equivalent in the wide sense to the process (86).

Let $\Omega_{1}^{0}=R^{1}, \mathcal{F}_{1}^{0}=B\left(R^{1}\right)$, where $R^{1}$ is a real axis, $B\left(R^{1}\right)$ is a Borel $\sigma$-algebra on $R^{1}$. Let us put $\Omega_{i}^{0}=\Omega_{1}^{0}, \mathcal{F}_{i}^{0}=\mathcal{F}_{1}^{0}, i=\overline{1, N}$, and let us construct the direct product of the measurable spaces $\left\{\Omega_{i}^{0}, \mathcal{F}_{i}^{0}\right\}, i=\overline{1, N}$. Denote $\Omega=\prod_{i=1}^{N} \Omega_{i}^{0}$. Under the $\sigma$-algebra $\mathcal{F}$ on $\Omega$, we understand the minimal $\sigma$-algebra generated by sets $\prod_{i=1}^{N} G_{i}, G_{i} \in \mathcal{F}_{i}^{0}$. On the measurable space $\{\Omega, \mathcal{F}\}$, under the filtration $\mathcal{F}_{n}$ we understand the minimal $\sigma$-algebra, generated by sets $\prod_{i=1}^{N} G_{i}, G_{i} \in \mathcal{F}_{i}^{0}$, where $G_{i}=\Omega_{i}^{0}$ for $i>n$. Suppose that the points $t_{0}=0, t_{1}, t_{2}, \cdots, t_{N}=T$, belongs to $R_{1}^{+}$with $\Delta t=t_{i}-t_{i-1}>0$ not depending on the index $i$. Let us consider the probability space $\{\Omega, \mathcal{F}, P\}$, where $P=\prod_{i=1}^{N} P_{i}^{0}, P_{i}^{0}=P_{1}^{0}, \quad i=\overline{1, N}$, 


$$
P_{1}^{0}(A)=\frac{1}{[2 \pi \Delta t]^{1 / 2}} \int_{A} \mathrm{e}^{-\frac{y^{2}}{2 \Delta t}} \mathrm{~d} y, \quad A \in \mathcal{F}_{1}^{0} .
$$

On the probability space $\{\Omega, \mathcal{F}, P\}$, let us consider the evolution of risk asset given by the law

$$
S_{n}=S_{0} \prod_{s=1}^{n}\left(1+a_{s} \rho\left(y_{s}\right)\right), n=\overline{1, N},
$$

where $0 \leq a_{s} \leq 1, s=\overline{1, N}$, are the same constants, that figure in the formula (86), $\quad \rho(y)=\mathrm{e}^{\sigma(d+y)}-1, \quad d=\frac{\left(\mu-\frac{\sigma^{2}}{2}-r\right) \Delta t}{\sigma}$.

On the probability space $\{\Omega, \mathcal{F}, P\}$, the random process given by the formula (88) is equivalent in the wide sense to the process (86), constructed above.

Described in Lemma 5 [1] the set of measures $Q$ on the probability space $\left\{\Omega_{i}^{0}, \mathcal{F}_{i}^{0}, P_{i}^{0}\right\}$, which are equivalent to the measure $P_{i}^{0}$ and such that $E^{Q} \rho\left(y_{i}\right)=0$, we denote by $M_{i}, i=\overline{1, N}$.

On the measurable space $\{\Omega, \mathcal{F}\}$, we introduce into consideration the set of measures $M_{0}^{N}$, described in Theorem 1 for $\eta_{i}\left(y_{i}\right)=\rho\left(y_{i}\right), i=\overline{1, N}$.

Theorem 9. On the measurable space $\{\Omega, \mathcal{F}\}$ with the filtration $\mathcal{F}_{n}, n=\overline{0, N}$, on it, let the risk asset evolution is given by the formula (88). For the nonnegative payoff function $f\left(S_{N}\right)$, satisfying the condition $\sup _{N}^{Q} f\left(S_{N}\right)<\infty$, the fair price of super-hedge is given by the formula

$$
\begin{aligned}
& \sup _{Q \in M_{0}^{N}} E^{Q} f\left(S_{N}\right) \\
& =\sup _{y_{i}^{1} \leq-d, y_{i}^{2}>-d, i=1, N} \sum_{i_{1}=1, \cdots, i_{N}=1}^{2} f\left(S_{0} \prod_{s=1}^{N}\left(1+a_{s} \rho\left(y_{s}^{i_{s}}\right)\right)\right) \\
& \quad \times \prod_{s=1}^{N} \frac{\left|\mathrm{e}^{\sigma\left(d+y_{s}^{i_{s}+1}\right)}-1\right|}{\mid \mathrm{e}^{\sigma\left(d+y_{s}^{i_{s}+1}\right)}-\mathrm{e}^{\sigma\left(d+y_{s}^{i_{s}}\right) \mid},}
\end{aligned}
$$

where we put $y_{s}^{3}=y_{s}^{1}$.

Proof. The proof of Theorem 9 follows directly from Theorem 6 .

Theorem 10. Suppose that the evolution of risk asset is given by the formula (88). If the nonnegative payoff function $f(x), x \in[0, \infty)$, satisfies the conditions:

1) $f(0)=0, f(x) \leq a x, \lim _{x \rightarrow \infty} \frac{f(x)}{x}=a, a>0$, then

$$
f\left(S_{0} \prod_{i=1}^{N}\left(1-a_{i}\right)\right)+a S_{0}\left(1-\prod_{i=1}^{N}\left(1-a_{i}\right)\right) \leq \sup _{Q \in M_{0}^{N}} E^{P} f\left(S_{N}\right) \leq a S_{0} .
$$

If, in addition, the nonnegative payoff function $f(x)$ is a convex down one, then

$$
\inf _{P \in M_{0}^{N}} E^{P} f\left(S_{N}\right)=f\left(S_{0}\right),
$$


where $M_{0}^{N}$ is the set of equivalent martingale measures for the evolution of risk asset $S_{n}, n=\overline{0, N}$.

Proof. As before,

$$
\begin{aligned}
& a S_{0} \geq \sup _{Q \in M_{0}^{N}} \int_{\Omega} f\left(S_{N}\right) \mathrm{d} Q \\
& =\sup _{y_{i}^{1} \leq-d, y_{i}^{2}>-d, i=1, N} \sum_{i_{1}=1, \cdots, i_{N}=1}^{2} f\left(S_{0} \prod_{s=1}^{N}\left(1+a_{s} \rho\left(y_{s}^{i_{s}}\right)\right)\right) \\
& \times \prod_{s=1}^{N} \frac{\left|\mathrm{e}^{\sigma\left(d+y_{s}^{i_{s}+1}\right)}-1\right|}{\left|\mathrm{e}^{\sigma\left(d+y_{s}^{i_{s}+1}\right)}-\mathrm{e}^{\sigma\left(d+y_{s}^{i_{s}}\right)}\right|} \\
& \geq \sum_{i_{1}=1, \cdots, i_{N-1}=1}^{2} \prod_{s=1}^{N-1} \frac{\left|\mathrm{e}^{\sigma\left(d+y_{s}^{i_{s}+1}\right)}-1\right|}{\mathrm{e}^{\sigma\left(d+y_{s}^{i_{s}+1}\right)}-\mathrm{e}^{\sigma\left(d+y_{s}^{i_{s}}\right)} \mid} \\
& \times \lim _{y_{N}^{1} \rightarrow-\infty, y_{N}^{2} \rightarrow \infty}\left[f\left(S_{0} \prod_{s=1}^{N-1}\left(1+a_{s} \rho\left(y_{s}^{i_{s}}\right)\right)\left(1+a_{N} \rho\left(y_{N}^{1}\right)\right)\right) \frac{\mathrm{e}^{\sigma\left(d+y_{N}^{2}\right)}-1}{\mathrm{e}^{\sigma\left(d+y_{N}^{2}\right)}-\mathrm{e}^{\sigma\left(d+y_{N}^{1}\right)}}\right. \\
& \left.+f\left(S_{0} \prod_{s=1}^{N-1}\left(1+a_{s} \rho\left(y_{s}^{i_{s}}\right)\right)\left(1+a_{N} \rho\left(y_{N}^{2}\right)\right)\right) \frac{1-\mathrm{e}^{\sigma\left(d+y_{N}^{1}\right)}}{\mathrm{e}^{\sigma\left(d+y_{N}^{2}\right)}-\mathrm{e}^{\sigma\left(d+y_{N}^{1}\right)}}\right] \\
& =\sum_{i_{1}=1, \cdots, i_{N-1}=1}^{2} \prod_{s=1}^{N-1} \frac{\left|\mathrm{e}^{\sigma\left(d+y_{s}^{i_{s}+1}\right)}-1\right|}{\mathrm{e}^{\sigma\left(d+y_{s}^{i_{s}+1}\right)}-\mathrm{e}^{\sigma\left(d+y_{s}^{i_{s}}\right)} \mid} \\
& \times\left[f\left(S_{0} \prod_{s=1}^{N-1}\left(1+a_{s} \rho\left(y_{s}^{i_{s}}\right)\right)\left(1-a_{N}\right)\right)+a_{N} a S_{0} \prod_{s=1}^{N-1}\left(1+a_{s} \rho\left(y_{s}^{i_{s}}\right)\right)\right] \\
& =\sum_{i_{1}=1, \cdots, i_{N-1}=1}^{2} \prod_{s=1}^{N-1} \frac{\left|\mathrm{e}^{\sigma\left(d+y_{s}^{i_{s}+1}\right)}-1\right|}{\left|\mathrm{e}^{\sigma\left(d+y_{s}^{i_{s}+1}\right)}-\mathrm{e}^{\sigma\left(d+y_{s}^{i_{s}}\right)}\right|} \\
& \times f\left(S_{0}\left(1-a_{N}\right) \prod_{s=1}^{N-1}\left(1+a_{s} \rho\left(y_{s}^{i_{s}}\right)\right)\right)+a_{N} a S_{0} .
\end{aligned}
$$

Applying $(N-1)$ times the inequality (92) we obtain

$$
\begin{aligned}
\sup _{Q \in M_{0}^{N}} \int_{\Omega} f\left(S_{N}\right) \mathrm{d} Q & \geq f\left(S_{0} \prod_{i=1}^{N}\left(1-a_{i}\right)\right)+a S_{0} \sum_{i=1}^{N} a_{i} \prod_{s=i+1}^{N}\left(1-a_{s}\right) \\
& =f\left(S_{0} \prod_{i=1}^{N}\left(1-a_{i}\right)\right)+a S_{0}\left(1-\prod_{i=1}^{N}\left(1-a_{i}\right)\right) .
\end{aligned}
$$

Let us prove the equality (91). Using Jensen inequality we obtain

$$
\inf _{P \in M_{0}^{N}} E^{P} f\left(S_{N}\right) \geq f\left(S_{0}\right) .
$$

It is evident the inequality 


$$
\begin{aligned}
& {\left[f\left(S_{0}\left(1+a_{s} \rho\left(y_{s}^{1}\right)\right)\right) \frac{\mathrm{e}^{\sigma\left(d+y_{s}^{2}\right)}-1}{\mathrm{e}^{\sigma\left(d+y_{s}^{2}\right)}-\mathrm{e}^{\sigma\left(d+y_{s}^{1}\right)}}\right.} \\
& \left.+f\left(S_{0}\left(1+a_{s} \rho\left(y_{s}^{2}\right)\right)\right) \frac{1-e^{\sigma\left(d+y_{s}^{1}\right)}}{\mathrm{e}^{\sigma\left(d+y_{s}^{2}\right)}-\mathrm{e}^{\sigma\left(d+y_{s}^{1}\right)}}\right] \geq \inf _{P \in M_{0}^{N}} E^{P} f\left(S_{N}\right) .
\end{aligned}
$$

Putting in the inequality (95) $y_{s}^{1}=-d$ we obtain the inverse inequality.

Theorem 11. Suppose that the evolution of risk asset is given by the formula (88). If the nonnegative payoff function $f(x), x \in[0, \infty)$, satisfies the conditions:

1) $f(0)=K, f(x) \leq K$, then

$$
f\left(S_{0} \prod_{i=1}^{N}\left(1-a_{i}\right)\right) \leq \sup _{P \in M_{0}^{N}} E^{P} f\left(S_{N}\right) \leq K .
$$

If, in addition, the nonnegative payoff function $f(x)$ is a convex down one, then

$$
\inf _{P \in M_{0}^{N}} E^{P} f\left(S_{N}\right)=f\left(S_{0}\right),
$$

where $M_{0}^{N}$ is the set of equivalent martingale measures for the evolution of risk asset $S_{n}, n=\overline{0, N}$.

Proof. Let us obtain the estimate from below. Really,

$$
\begin{aligned}
& K \geq \sup _{Q \in M_{0}^{N}} \int_{\Omega} f\left(S_{N}\right) \mathrm{d} Q \\
& =\sup _{y_{s}^{1} \leq-d, y_{s}^{2}>-d, s=1, N} \sum_{i_{1}=1, \cdots, i_{N}=1}^{2} f\left(S_{0} \prod_{s=1}^{N}\left(1+a_{s} \rho\left(y_{s}^{i_{s}}\right)\right)\right) \\
& \times \prod_{s=1}^{N} \frac{\left|\mathrm{e}^{\sigma\left(d+y_{s}^{i_{s}+1}\right)}-1\right|}{\mathrm{e}^{\sigma\left(d+y_{s}^{i_{s}+1}\right)}-\mathrm{e}^{\sigma\left(d+y_{s}^{i_{s}}\right)} \mid} \\
& \geq \sum_{i_{1}=1, \cdots, i_{N-1}=1}^{2} \prod_{s=1}^{N-1} \frac{\left|\mathrm{e}^{\sigma\left(d+y_{s}^{i_{s}+1}\right)}-1\right|}{\mathrm{e}^{\sigma\left(d+y_{s}^{i_{s}+1}\right)}-\mathrm{e}^{\sigma\left(d+y_{s}^{i_{s}}\right)} \mid} \\
& \times \lim _{y_{N}^{1} \rightarrow-\infty, y_{N}^{2} \rightarrow \infty}\left[f\left(S_{0} \prod_{s=1}^{N-1}\left(1+a_{s} \rho\left(y_{s}^{i_{s}}\right)\right)\left(1+a_{N} \rho\left(y_{N}^{1}\right)\right)\right) \frac{\mathrm{e}^{\sigma\left(d+y_{N}^{2}\right)}-1}{\mathrm{e}^{\sigma\left(d+y_{N}^{2}\right)}-\mathrm{e}^{\sigma\left(d+y_{N}^{1}\right)}}\right. \\
& \left.+f\left(S_{0} \prod_{s=1}^{N-1}\left(1+a_{s} \rho\left(y_{s}^{i_{s}}\right)\right)\left(1+a_{N} \rho\left(y_{N}^{2}\right)\right)\right) \frac{1-\mathrm{e}^{\sigma\left(d+y_{N}^{1}\right)}}{\mathrm{e}^{\sigma\left(d+y_{N}^{2}\right)}-\mathrm{e}^{\sigma\left(d+y_{N}^{1}\right)}}\right] \\
& =\sum_{i_{1}=1, \cdots, i_{N-1}=1}^{2} \prod_{s=1}^{N-1} \frac{\left|\mathrm{e}^{\sigma\left(d+y_{s}^{i_{s}+1}\right)}-1\right|}{\mathrm{e}^{\sigma\left(d+y_{s}^{i_{s}+1}\right)}-\mathrm{e}^{\sigma\left(d+y_{s}^{i_{s}}\right)} \mid} \\
& \times f\left(S_{0}\left(1-a_{N}\right) \prod_{s=1}^{N-1}\left(1+a_{s} \rho\left(y_{s}^{i_{s}}\right)\right)\right) .
\end{aligned}
$$


Applying $(N-1)$ times the inequality (98) we obtain the needed inequality

$$
\sup _{Q \in M_{0}^{N}} \int_{\Omega} f\left(S_{N}\right) \mathrm{d} Q \geq f\left(S_{0} \prod_{i=1}^{N}\left(1-a_{i}\right)\right) .
$$

Let us prove the equality (97). Using Jensen inequality we obtain

$$
\inf _{P \in M_{0}^{N}} E^{P} f\left(S_{N}\right) \geq f\left(S_{0}\right) .
$$

It is evident the inequality

$$
\begin{aligned}
& {\left[f\left(S_{0}\left(1+a_{s} \rho\left(y_{s}^{1}\right)\right)\right) \frac{\mathrm{e}^{\sigma\left(d+y_{s}^{2}\right)}-1}{\mathrm{e}^{\sigma\left(d+y_{s}^{2}\right)}-\mathrm{e}^{\sigma\left(d+y_{s}^{1}\right)}}\right.} \\
& \left.+f\left(S_{0}\left(1+a_{s} \rho\left(y_{s}^{2}\right)\right)\right) \frac{1-\mathrm{e}^{\sigma\left(d+y_{s}^{1}\right)}}{\mathrm{e}^{\sigma\left(d+y_{s}^{2}\right)}-\mathrm{e}^{\sigma\left(d+y_{s}^{1}\right)}}\right] \geq \inf _{P \in M_{0}^{N}} E^{P} f\left(S_{N}\right) .
\end{aligned}
$$

Putting in the inequality (101) $y_{s}^{1}=-d$ we obtain the inverse inequality.

Theorem 12. On the measurable space $\{\Omega, \mathcal{F}\}$ with the filtration $\mathcal{F}_{n}$, $n=\overline{0, N}$, on it, let the discount risk asset evolution is given by the formula (88) with $0 \leq a_{i} \leq 1, i=\overline{1, N}$, For the payoff function $f(x)=(x-K)^{+}, x \in(0, \infty)$, $K>0$, the fair price of super-hedge is given by the formula

$$
\sup _{Q \in M_{0}^{N}} E^{Q} f\left(S_{N}\right)= \begin{cases}\left(S_{0}-K\right)^{+}, & \text {if } S_{0} \prod_{i=1}^{N}\left(1-a_{i}\right) \geq K, \\ S_{0}\left(1-\prod_{i=1}^{N}\left(1-a_{i}\right)\right), & \text { if } S_{0} \prod_{i=1}^{N}\left(1-a_{i}\right)<K .\end{cases}
$$

If $S_{0} \prod_{i=1}^{N}\left(1-a_{i}\right) \geq K$, then the set of non arbitrage prices coincides with the point $\left(S_{0}-K\right)^{+}$, in case if $S_{0}\left(1-\prod_{i=1}^{N}\left(1-a_{i}\right)\right)<K$ the set of non arbitrage prices coincides with the interval $\left[\left(S_{0}-K\right)^{+}, S_{0}\left(1-\prod_{i=1}^{N}\left(1-a_{i}\right)\right)\right]$.

Proof. Let us introduce the denotations

$$
\begin{aligned}
& I_{N}=\sum_{i_{1}=1, \cdots, i_{N}=1}^{2} f\left(S_{0} \prod_{s=1}^{N}\left(1+a_{s} \rho\left(y_{s}^{i_{s}}\right)\right)\right) \prod_{s=1}^{N} \frac{\left|\mathrm{e}^{\sigma\left(d+y_{s}^{i_{s}+1}\right)}-1\right|}{\left|\mathrm{e}^{\sigma\left(d+y_{s}^{i_{s}+1}\right)}-\mathrm{e}^{\sigma\left(d+y_{s}^{i_{s}}\right)}\right|}, \\
& I_{N}^{1}=\sum_{i_{1}=1, \cdots, i_{N}=1}^{2} f_{1}\left(S_{0} \prod_{s=1}^{N}\left(1+a_{s} \rho\left(y_{s}^{i_{s}}\right)\right)\right) \prod_{s=1}^{N} \frac{\left|\mathrm{e}^{\sigma\left(d+y_{s}^{i_{s}+1}\right)}-1\right|}{\left|\mathrm{e}^{\sigma\left(d+y_{s}^{i_{s}+1}\right)}-\mathrm{e}^{\sigma\left(d+y_{s}^{i_{s}}\right)}\right|}, \\
& I_{N}^{0}=\sup _{y_{s}^{1} \leq-d, y_{s}^{2}>-d, s=1, \bar{N}} \sum_{i_{1}=1, \cdots, i_{N}=1}^{2} f\left(S_{0} \prod_{s=1}^{N}\left(1+a_{s} \rho\left(y_{s}^{i_{s}}\right)\right)\right) \\
& \times \prod_{s=1}^{N} \frac{\left|\mathrm{e}^{\sigma\left(d+y_{s}^{i_{s}+1}\right)}-1\right|}{\left|\mathrm{e}^{\sigma\left(d+y_{s}^{i_{s}+1}\right)}-\mathrm{e}^{\sigma\left(d+y_{s}^{i_{s}}\right)}\right|}
\end{aligned}
$$


where we put $f_{1}(x)=(K-x)^{+}$.

Let us estimate from above the value

$$
I_{N}=\sum_{i_{1}=1, \cdots, i_{N}=1}^{2} f\left(S_{0} \prod_{s=1}^{N}\left(1+a_{s} \rho\left(y_{s}^{i_{s}}\right)\right)\right) \prod_{s=1}^{N} \frac{\left|\mathrm{e}^{\sigma\left(d+y_{s}^{i_{s}+1}\right)}-1\right|}{\left|\mathrm{e}^{\sigma\left(d+y_{s}^{i_{s}+1}\right)}-\mathrm{e}^{\sigma\left(d+y_{s}^{i_{s}}\right)}\right|} .
$$

For this we use the equality

$$
\begin{aligned}
& \sum_{i_{1}=1, \cdots, i_{N}=1}^{2} f\left(S_{0} \prod_{s=1}^{N}\left(1+a_{s} \rho\left(y_{s}^{i_{s}}\right)\right)\right) \prod_{s=1}^{N} \frac{\left|\mathrm{e}^{\sigma\left(d+y_{s}^{i_{s}+1}\right)}-1\right|}{\mathrm{e}^{\sigma\left(d+y_{s}^{i_{s}+1}\right)}-\mathrm{e}^{\sigma\left(d+y_{s}^{i_{s}}\right)} \mid} \\
& =\sum_{i_{1}=1, \cdots, i_{N}=1}^{2} f_{1}\left(S_{0} \prod_{s=1}^{N}\left(1+a_{s} \rho\left(y_{s}^{i_{s}}\right)\right)\right) \prod_{s=1}^{N} \frac{\left|\mathrm{e}^{\sigma\left(d+y_{s}^{i_{s}+1}\right)}-1\right|}{\mathrm{e}^{\sigma\left(d+y_{s}^{i_{s}+1}\right)}-\mathrm{e}^{\sigma\left(d+y_{s}^{i_{s}}\right)} \mid}+S_{0}-K \\
& =I_{N}^{1}+S_{0}-K,
\end{aligned}
$$

which follows from the identity: $f(x)=f_{1}(x)+x-K, \quad x \geq 0$. Since

$$
f_{1}\left(S_{0} \prod_{s=1}^{N}\left(1+a_{s} \rho\left(y_{s}^{i_{s}}\right)\right)\right) \leq f_{1}\left(S_{0} \prod_{s=1}^{N}\left(1-a_{s}\right)\right)
$$

we obtain the inequality

$$
I_{N} \leq S_{0}-K+f_{1}\left(S_{0} \prod_{s=1}^{N}\left(1-a_{s}\right)\right) .
$$

From the inequality (109) we have

$$
\begin{aligned}
I_{N}^{0} \leq & S_{0}-K+f_{1}\left(S_{0} \prod_{s=1}^{N}\left(1-a_{s}\right)\right) \\
& = \begin{cases}\left(S_{0}-K\right)^{+}, & \text {if } S_{0} \prod_{i=1}^{N}\left(1-a_{i}\right) \geq K, \\
S_{0}\left(1-\prod_{i=1}^{N}\left(1-a_{i}\right)\right), & \text { if } S_{0} \prod_{i=1}^{N}\left(1-a_{i}\right)<K .\end{cases}
\end{aligned}
$$

Due to the inequality (90) of Theorem 10

$$
\begin{aligned}
& \sup _{y_{i}^{1} \leq-d, y_{i}^{2}>-d, i=1, N} \sum_{i_{1}=1, \cdots, i_{N}=1}^{2} f\left(S_{0} \prod_{s=1}^{N}\left(1+a_{s} \rho\left(y_{s}^{i_{s}}\right)\right)\right) \prod_{s=1}^{N} \frac{\left|\mathrm{e}^{\sigma\left(d+y_{s}^{i_{s}+1}\right)}-1\right|}{\mathrm{e}^{\sigma\left(d+y_{s}^{i_{s}+1}\right)}-\mathrm{e}^{\sigma\left(d+y_{s}^{i_{s}}\right)} \mid} \\
& \geq f\left(S_{0} \prod_{i=1}^{N}\left(1-a_{i}\right)\right)+S_{0}\left(1-\prod_{i=1}^{N}\left(1-a_{i}\right)\right)
\end{aligned}
$$

and the inequality

$$
\begin{aligned}
& \sup _{y_{i}^{1} \leq-d, y_{i}^{2}>-d, i=\overline{1, N} i_{1}=1, \cdots, i_{N}=1} \sum^{2} f\left(S_{0} \prod_{s=1}^{N}\left(1+a_{s} \rho\left(y_{s}^{i_{s}}\right)\right)\right) \\
& \times \prod_{s=1}^{N} \frac{\left|\mathrm{e}^{\sigma\left(d+y_{s}^{i_{s}+1}\right)}-1\right|}{\left|\mathrm{e}^{\sigma\left(d+y_{s}^{i_{s}+1}\right)}-\mathrm{e}^{\sigma\left(d+y_{s}^{i_{s}}\right)}\right|} \geq\left(S_{0}-K\right)^{+},
\end{aligned}
$$


which follows from Jensen inequality, we have

$$
\begin{aligned}
I_{N}^{0} \geq & \max \left\{\left(S_{0}-K\right)^{+}, f\left(S_{0} \prod_{i=1}^{N}\left(1-a_{i}\right)\right)+S_{0}\left(1-\prod_{i=1}^{N}\left(1-a_{i}\right)\right)\right\} \\
& = \begin{cases}\left(S_{0}-K\right)^{+}, & \text {if } S_{0} \prod_{i=1}^{N}\left(1-a_{i}\right) \geq K, \\
S_{0}\left(1-\prod_{i=1}^{N}\left(1-a_{i}\right)\right), & \text { if } S_{0} \prod_{i=1}^{N}\left(1-a_{i}\right)<K .\end{cases}
\end{aligned}
$$

This proves Theorem 12 .

Theorem 13. On the measurable space $\{\Omega, \mathcal{F}\}$ with the filtration $\mathcal{F}_{n}$, $n=\overline{0, N}$, on it, let the discount risk asset evolution is given by the formula (88), with $0 \leq a_{i} \leq 1, i=\overline{1, N}$. For the payoff function $f_{1}(x)=(K-x)^{+}, x \in(0, \infty)$, $K>0$, the fair price of super-hedge is given by the formula

$$
\sup _{Q \in M_{0}^{N}} E^{Q} f_{1}\left(S_{N}\right)=f_{1}\left(S_{0} \prod_{i=1}^{N}\left(1-a_{i}\right)\right) .
$$

The set of non arbitrage prices coincides with the set

$$
\left[\left(K-S_{0}\right)^{+}, f_{1}\left(S_{0} \prod_{i=1}^{N}\left(1-a_{i}\right)\right)\right] \text {. }
$$

Proof. The inequality

$$
\begin{aligned}
& \sum_{i_{1}=1, \cdots, i_{N}=1}^{2} f_{1}\left(S_{0} \prod_{s=1}^{N}\left(1+a_{s} \rho\left(y_{s}^{i_{s}}\right)\right)\right) \prod_{s=1}^{N} \frac{\left|\mathrm{e}^{\sigma\left(d+y_{s}^{i_{s}+1}\right)}-1\right|}{\left|\mathrm{e}^{\sigma\left(d+y_{s}^{i_{s}+1}\right)}-\mathrm{e}^{\sigma\left(d+y_{s}^{i_{s}}\right)}\right|} \\
& \leq \sum_{i_{1}=1, \cdots, i_{N}=1}^{2} f_{1}\left(S_{0} \prod_{s=1}^{N}\left(1-a_{s}\right)\right) \prod_{s=1}^{N} \frac{\mathrm{e}^{\sigma\left(d+y_{s}^{2}\right)}-1}{\mathrm{e}^{\sigma\left(d+y_{s}^{2}\right)}-\mathrm{e}^{\sigma\left(d+y_{s}^{1}\right)}} \\
& =f_{1}\left(S_{0} \prod_{i=1}^{N}\left(1-a_{i}\right)\right)
\end{aligned}
$$

is true. Taking into account the inequality (96) of Theorem 11 we prove Theorem 13.

Theorem 14. On the measurable space $\{\Omega, \mathcal{F}\}$ with the filtration $\mathcal{F}_{n}$, $n=\overline{0, N}$, on it, let the discount risk asset evolution is given by the formula (88) with $0 \leq a_{i} \leq 1, i=\overline{1, N}$. For the payoff function

$f_{1}\left(S_{0}, S_{1}, \cdots, S_{N}\right)=\left(K-\frac{\sum_{i=0}^{N} S_{i}}{N+1}\right)^{+}, K>0$, the fair price of super-hedge is given by the formula

$$
\sup _{Q \in M_{0}^{N}} E^{Q} f_{1}\left(S_{0}, S_{1}, \cdots, S_{N}\right)=\left(K-\frac{S_{0} \sum_{i=0}^{N} \prod_{s=1}^{i}\left(1-a_{s}\right)}{N+1}\right)^{+} .
$$

The set of non arbitrage prices coincides with the set 
$\left[\left(K-S_{0}\right)^{+},\left(K-\frac{S_{0} \sum_{i=0}^{N} \prod_{s=1}^{i}\left(1-a_{s}\right)}{N+1}\right)^{+}\right]$, if $K>\frac{S_{0} \sum_{i=0}^{N} \prod_{s=1}^{i}\left(1-a_{s}\right)}{N+1}$. For
$K \leq \frac{S_{0} \sum_{i=0}^{N} \prod_{s=1}^{i}\left(1-a_{s}\right)}{N+1}$ the set of non arbitrage prices coincides with the point 0.

Proof. It is evident that

$$
\begin{aligned}
& \sup _{y_{i}^{1} \leq-d, y_{i}^{2}>-d, i=\overline{1, N}{ }_{i_{1}=1, \cdots, i_{N}=1}} f_{1}\left(S_{0}, S_{0}\left(1+a_{1} \rho\left(y_{1}^{i_{1}}\right)\right), \cdots, S_{0} \prod_{s=1}^{N}\left(1+a_{s} \rho\left(y_{s}^{i_{s}}\right)\right)\right) \\
& \times \prod_{s=1}^{N} \frac{\left|\mathrm{e}^{\sigma\left(d+y_{s}^{i_{s}+1}\right)}-1\right|}{\mathrm{e}^{\sigma\left(d+y_{s}^{i_{s}+1}\right)}-\mathrm{e}^{\sigma\left(d+y_{s}^{i_{s}}\right) \mid}} \\
& \geq \lim _{y_{s}^{1} \rightarrow-\infty, y_{s}^{2} \rightarrow \infty, s=\overline{1, N}} f_{1}\left(S_{0}, S_{0}\left(1+a_{1} \rho\left(y_{1}^{1}\right)\right), \cdots, S_{0} \prod_{s=1}^{N}\left(1+a_{s} \rho\left(y_{s}^{1}\right)\right)\right) \\
& \times \prod_{s=1}^{N} \frac{\mathrm{e}^{\sigma\left(d+y_{s}^{2}\right)}-1}{\mathrm{e}^{\sigma\left(d+y_{s}^{2}\right)}-\mathrm{e}^{\sigma\left(d+y_{s}^{1}\right)}} \\
& =f_{1}\left(S_{0}, S_{0}\left(1-a_{1}\right), \cdots, S_{0} \prod_{s=1}^{N}\left(1-a_{s}\right)\right)=\left(K-\frac{S_{0} \sum_{i=0}^{N} \prod_{s=1}^{i}\left(1-a_{s}\right)}{N+1}\right)^{+} .
\end{aligned}
$$

Let us prove the inverse inequality. We have

$$
\begin{aligned}
& \sum_{i_{1}=1, \cdots, i_{N}=1}^{2} f_{1}\left(S_{0}, S_{0}\left(1+a_{1} \rho\left(y_{1}^{i_{1}}\right)\right), \cdots, S_{0} \prod_{s=1}^{N}\left(1+a_{s} \rho\left(y_{s}^{i_{s}}\right)\right)\right) \\
& \times \frac{\left|\mathrm{e}^{\sigma\left(d+y_{s}^{s_{s}+1}\right)}-1\right|}{\mid \mathrm{e}^{\sigma\left(d+y_{s}^{i_{s}+1}\right)}-\mathrm{e}^{\sigma\left(d+y_{s}^{i_{s}}\right) \mid}} \\
& \leq \sum_{i_{1}=1, \cdots, i_{N}=1}^{2} f_{1}\left(S_{0}, S_{0}\left(1-a_{1}\right), \cdots, S_{0} \prod_{s=1}^{N}\left(1-a_{s}\right)\right) \prod_{s=1}^{N} \frac{\mathrm{e}^{\sigma\left(d+y_{s}^{2}\right)}-1}{\mathrm{e}^{\sigma\left(d+y_{s}^{2}\right)}-\mathrm{e}^{\sigma\left(d+y_{s}^{1}\right)}} \\
& =f_{1}\left(S_{0}, S_{0}\left(1-a_{1}\right), \cdots, S_{0} \prod_{s=1}^{N}\left(1-a_{s}\right)\right)=\left(K-\frac{S_{0} \sum_{i=0}^{N} \prod_{s=1}^{N}\left(1-a_{s}\right)}{N+1}\right)^{+} .
\end{aligned}
$$

Therefore,

$$
\begin{aligned}
& \sup _{y_{i}^{1} \leq-d, y_{i}^{2}>-d, i=1, N} \sum_{i_{1}=1, \cdots, i_{N}=1}^{2} f_{1}\left(S_{0}, S_{0}\left(1+a_{1} \rho\left(y_{1}^{i_{1}}\right)\right), \cdots, S_{0} \prod_{s=1}^{N}\left(1+a_{s} \rho\left(y_{s}^{i_{s}}\right)\right)\right) \\
& \times \prod_{s=1}^{N} \frac{\left|\mathrm{e}^{\sigma\left(d+y_{s}^{i_{s}+1}\right)}-1\right|}{\mathrm{e}^{\sigma\left(d+y_{s}^{i_{s}+1}\right)}-\mathrm{e}^{\sigma\left(d+y_{s}^{i_{s}}\right)} \mid} \leq\left(K-\frac{S_{0} \sum_{i=0}^{N} \prod_{s=1}^{i}\left(1-a_{s}\right)}{N+1}\right)^{+} .
\end{aligned}
$$


The inequalities (117), (119) prove Theorem 14.

Theorem 15. On the measurable space $\{\Omega, \mathcal{F}\}$ with the filtration $\mathcal{F}_{n}$, $n=\overline{0, N}$, on it, let the discount risk asset evolution is given by the formula (88) with $0 \leq a_{i} \leq 1, i=\overline{1, N}$. For the payoff function $f\left(S_{0}, S_{1}, \cdots, S_{N}\right)=\left(\frac{\sum_{i=0}^{N} S_{i}}{N+1}-K\right)^{+}, K>0$, the fair price of super-hedge is given by the formula

$$
\begin{aligned}
& \sup _{Q \in M_{0}^{N}} E^{Q} f\left(S_{0}, S_{1}, \cdots, S_{N}\right) \\
& = \begin{cases}\left(S_{0}-K\right)^{+}, & \text {if } \frac{S_{0} \sum_{i=0}^{N} \prod_{s=1}^{i}\left(1-a_{i}\right)}{N+1} \geq K, \\
S_{0}\left(1-\frac{\sum_{i=0}^{N} \prod_{s=1}^{i}\left(1-a_{s}\right)}{N+1}\right), & \text { if } S_{0} \frac{\sum_{i=0}^{N} \prod_{s=1}^{i}\left(1-a_{s}\right)}{N+1}<K .\end{cases}
\end{aligned}
$$

If $\frac{S_{0} \sum_{i=0}^{N} \prod_{s=1}^{i}\left(1-a_{i}\right)}{N+1} \geq K$, then the set of non arbitrage prices coincides with the point $\left(S_{0}-K\right)^{+}$, in case if $S_{0} \frac{\sum_{i=0}^{N} \prod_{s=1}^{i}\left(1-a_{s}\right)}{N+1}<K$ the set of non arbitrage prices coincides with the interval $\left[\left(S_{0}-K\right)^{+}, S_{0}\left(1-\frac{\sum_{i=0}^{N} \prod_{s=1}^{i}\left(1-a_{s}\right)}{N+1}\right)\right]$.

Proof. We have

$$
\begin{aligned}
& \sup _{y_{i}^{1} \leq-d, y_{i}^{2}>-d, i=1, N} \sum_{i_{1}=1, \cdots, i_{N}=1}^{2} f\left(S_{0}, S_{0}\left(1+a_{1} \rho\left(y_{1}^{i_{1}}\right)\right), \cdots, S_{0} \prod_{s=1}^{N}\left(1+a_{s} \rho\left(y_{s}^{i_{s}}\right)\right)\right) \\
& \times \frac{\left|\mathrm{e}^{\sigma\left(d+y_{s}^{i_{s}+1}\right)}-1\right|}{\left|\mathrm{e}^{\sigma\left(d+y_{s}^{i_{s}+1}\right)}-\mathrm{e}^{\sigma\left(d+y_{s}^{i_{s}}\right) \mid}\right|} \\
& =\sup _{y_{i}^{1} \leq-d, y_{i}^{2}>-d, i=1, N} \sum_{i_{1}=1, \cdots, i_{N}=1}^{2} f_{1}\left(S_{0}, S_{0}\left(1+a_{1} \rho\left(y_{1}^{i_{1}}\right)\right), \cdots, S_{0} \prod_{s=1}^{N}\left(1+a_{s} \rho\left(y_{s}^{i_{s}}\right)\right)\right) \\
& \times \prod_{s=1}^{N} \frac{\left|\mathrm{e}^{\sigma\left(d+y_{s}^{i_{s}+1}\right)}-1\right|}{\mathrm{e}^{\sigma\left(d+y_{s}^{i_{s}+1}\right)}-\mathrm{e}^{\sigma\left(d+y_{s}^{i_{s}}\right)}+S_{0}-K} \\
& =\left(S_{0}-K\right)+\left(\begin{array}{c}
S_{0} \sum_{i=0}^{N} \prod_{s=1}^{i}\left(1-a_{s}\right) \\
N+1
\end{array}\right)^{+}
\end{aligned}
$$




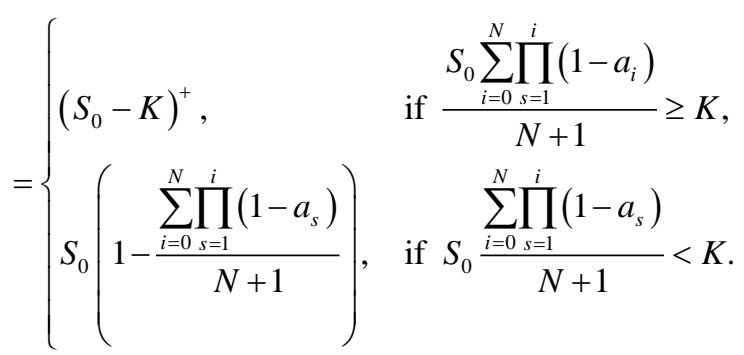

In the formula (121) we introduced the denotation

$$
f_{1}\left(S_{0}, S_{1}, \cdots, S_{N}\right)=\left(K-\frac{\sum_{i=0}^{N} S_{i}}{N+1}\right)^{+}
$$

The proof of Theorem 15 follows from the equality (121).

\section{Characteristic of the Random Processes Built on the Discrete Geometric Brownian Motion}

On the probability space $\{\Omega, \mathcal{F}, P\}$, with every sequence real numbers $a_{1}, a_{2}, \cdots, a_{n}, \cdots, a_{N}, \quad 0 \leq a_{i} \leq 1, \quad i=\overline{1, N}$, let us connect the random process

$$
S_{n}=S_{0} \prod_{s=1}^{n}\left(1+a_{s} \rho\left(y_{s}\right)\right), n=\overline{1, N}, \quad S_{0}>0 .
$$

where $0 \leq a_{s} \leq 1, \quad s=\overline{1, N}$, are constants, $\rho(y)=\mathrm{e}^{\sigma(d+y)}-1$,

$$
d=\frac{\left(\mu-\frac{\sigma^{2}}{2}-r\right) \Delta t}{\sigma} \text {. }
$$

Theorem 16. The random process (123) is a non homogeneous Markov process with the transition probability function for the $n$-th step

$$
P(n-1, x, n, A)=\int_{A} p(n-1, x, n, z) \mathrm{d} z, 0<a_{n} \leq 1, A \in B(0, \infty),
$$

where

$$
\begin{gathered}
p(n-1, x, n, z) \\
=\frac{\varphi_{n}(x, z)}{[2 \pi \Delta t]^{1 / 2} \sigma\left(z-x\left(1-a_{n}\right)\right)} \exp \left\{\frac{\left[\ln \left(\varphi_{n}(x, z) \frac{z-x\left(1-a_{n}\right)}{x a_{n}}\right)-\sigma d\right]^{2}}{2 \sigma^{2} \Delta t}\right\}, \\
0<a_{n} \leq 1, \quad \varphi_{n}(x, z)=\chi_{\left(x\left(1-a_{n}\right), \infty\right)}(z),
\end{gathered}
$$

and

$$
P(n-1, x, n, A)=\int_{A} \mathrm{~d} \mu_{x}(z)=\chi_{A}(x), \quad a_{n}=0,
$$

where $\mu_{x}(A)$ is an atomic measure which is concentrated at the point $x \in(0, \infty)$. 
Proof. Let us consider the case as $0<a_{n} \leq 1$. The transition probability function for one step is equal

$$
\begin{aligned}
& P(n-1, x, n, A)=P\left(S_{n} \in A \mid S_{n-1}=x\right)=P\left(x\left(1+a_{n} \rho\left(y_{n}\right)\right) \in A\right) \\
& =\frac{1}{(2 \pi \Delta t)^{1 / 2}} \int_{-\infty}^{\infty} \chi_{A}\left(x\left(1+a_{n}\left(\mathrm{e}^{\sigma(d+y)}-1\right)\right)\right) \mathrm{e}^{-\frac{y^{2}}{2 \Delta t}} \mathrm{~d} y .
\end{aligned}
$$

After the changing of variable $z=x\left(1+a_{n}\left(\mathrm{e}^{\sigma(d+y)}-1\right)\right)$ we obtain

$$
\mathrm{e}^{\sigma(d+y)}=\frac{z-\left(1-a_{n}\right) x}{x a_{n}} \chi_{\left(x\left(1-a_{n}\right), \infty\right)}(z),
$$

or

$$
y=\frac{1}{\sigma}\left[\ln \left(\varphi_{n}(x, z) \frac{z-x\left(1-a_{n}\right)}{x a_{n}}\right)-\sigma d\right] .
$$

For $z \geq\left(1-a_{n}\right) x$ we have

$$
\mathrm{e}^{\sigma(d+y)} \sigma \mathrm{d} y=\frac{\mathrm{d} z}{x a_{n}} .
$$

Substituting this into (127) we obtain

$$
\begin{aligned}
& P(n-1, x, n, A) \\
& =\frac{1}{[2 \pi \Delta t]^{1 / 2}} \int_{A} \frac{\varphi_{n}(x, z)}{\sigma\left(z-x\left(1-a_{n}\right)\right)} \exp \left\{\frac{\left[\ln \left(\varphi_{n}(x, z) \frac{z-x\left(1-a_{n}\right)}{x a_{n}}\right)-\sigma d\right]^{2}}{2 \sigma^{2} \Delta t}\right\} \mathrm{d} z \\
& \text { If } a_{n}=0 \text { then } P(n-1, x, n, A)=\chi_{A}(x) \text {, or } \\
& \qquad P(n-1, x, n, A)=\int_{A} 1 \mathrm{~d} \mu_{x}(z) .
\end{aligned}
$$

where we introduced the atomic measure $\mu_{x}(A), A \in B(0, \infty)$, concentrated at the point $x$.

Let us introduce the denotations $p_{i}(x, z)=p(i-1, x, i, z)$. First, suppose that all $1 \geq a_{i}>0, i=\overline{1, N}$, then the joint probability function is given by the formula

$$
\begin{aligned}
& P\left(S_{1} \in A_{1}, S_{2} \in A_{2}, \cdots, S_{N} \in A_{N}\right) \\
& =\int_{A_{1}} \cdots \int_{A_{N}} p_{1}\left(x, z_{1}\right) p_{2}\left(z_{1}, z_{2}\right) \cdots p_{N}\left(z_{N-1}, z_{N}\right) \mathrm{d} z_{1} \cdots \mathrm{d} z_{N} .
\end{aligned}
$$

If for some indexes $i_{1}, \cdots, i_{k}, a_{i_{s}}=0, s=\overline{1, k}$, then in this case the joint probability function

$$
\begin{aligned}
& P\left(S_{1} \in A_{1}, S_{2} \in A_{2}, \cdots, S_{N} \in A_{N}\right) \\
& =\int_{A_{1}} \cdots \int_{A_{N}} p_{1}\left(x, z_{1}\right) p_{2}\left(z_{1}, z_{2}\right) \cdots p_{N}\left(z_{N-1}, z_{N}\right) v\left(\mathrm{~d} z_{1} \cdots \mathrm{d} z_{N}\right),
\end{aligned}
$$

where the measure $v\left(A_{1} \cdots A_{N}\right)$ is a direct product of measures $\mu_{i}\left(A_{i}\right)$, $A_{i} \in B(0, \infty)$, where $\mu_{i}\left(A_{i}\right)$ is a Lebesgue measure for those $i$ for which 
$0<a_{i} \leq 1$ and $\mu_{i_{s}}\left(A_{i_{s}}\right)$ is an atomic measure which is concentrated at the point $z_{i_{s}-1}, s=\overline{1, k}$, under the case $a_{i_{s}}=0, s=\overline{1, k}$. Moreover, in the formula (134)

$$
\begin{aligned}
& p_{i}\left(z_{i-1}, z_{i}\right)= \frac{\varphi_{i}\left(z_{i-1}, z_{i}\right)}{[2 \pi \Delta t]^{1 / 2} \sigma\left(z_{i}-z_{i-1}\left(1-a_{i}\right)\right)} \\
& \times \exp \left\{\frac{\left[\ln \left(\varphi_{i}\left(z_{i-1}, z_{i}\right) \frac{z_{i}-z_{i-1}\left(1-a_{i}\right)}{z_{i-1} a_{i}}\right)-\sigma d\right]^{2}}{2 \sigma^{2} \Delta t}\right\}, \\
& 0<a_{i} \leq 1, \varphi_{i}\left(z_{i-1}, z_{i}\right)=\chi_{\left(z_{i-1}\left(1-a_{i}\right), \infty\right)}\left(z_{i}\right), \quad \sigma d=\left(\mu-\frac{\sigma^{2}}{2}-r\right) \Delta t, \\
& a_{i_{s}}=0, \quad p\left(z_{i_{s}-1}, z_{i_{s}}\right)=1, \quad s=\overline{1, k} .
\end{aligned}
$$

\section{Estimation of the Parameters of the Considered Random Process}

Suppose that $\left\{g_{i}\left(X_{N}\right)\right\}_{i=1}^{N}$ is a mapping from the set $[0,1]^{N}$ into itself, where $X_{N}=\left\{x_{1}, \cdots, x_{N}\right\}, 0 \leq x_{i} \leq 1, i=\overline{1, N}$. If $S_{0}, S_{1}, \cdots, S_{N}$ is a sample of the process (123) let us denote the order statistic $S_{(0)}, S_{(1)}, \cdots, S_{(N)}$ of this sample. Introduce also the denotation $g_{i}\left([S]_{N}\right)=g_{i}\left(\frac{S_{(0)}}{S_{(N)}}, \cdots, \frac{S_{(N-1)}}{S_{(N)}}\right), i=\overline{1, N}$.

Theorem 17. Suppose that $S_{0}, S_{1}, \cdots, S_{N}$ is a sample of the random process (123). Then for the parameters $a_{1}, \cdots, a_{N}$ the estimation

$$
\begin{gathered}
a_{1}=1-\tau_{0} \frac{S_{(0)}}{S_{0}} g_{1}\left([S]_{N}\right), \quad 0<\tau_{0} \leq 1, \\
a_{i}=1-\frac{g_{i}\left([S]_{N}\right)}{g_{i-1}\left([S]_{N}\right)}, \quad i=\overline{2, N},
\end{gathered}
$$

is valid, if for $g_{N}\left([S]_{N}\right)>0,[S]_{N} \in[0,1]^{N}$, the inequalities $g_{1}\left([S]_{N}\right) \geq g_{2}\left([S]_{N}\right) \geq \cdots \geq g_{N}\left([S]_{N}\right)$ are true. If $\tau_{0}=0$, then $a_{i}=1, i=\overline{1, N}$.

Proof. The estimation of the parameters $a_{1}, \cdots, a_{N}$ we do using the representation of random process $S_{n}, n=\overline{1, N}$. The smallest value of the random variable $S_{n}$ is equal $S_{0} \prod_{i=1}^{n}\left(1-a_{i}\right), n=\overline{1, N}$. Let us determine the parameters $a_{i}$ from the relations

$$
\begin{aligned}
& S_{0} \prod_{i=1}^{N}\left(1-a_{i}\right)=\tau g_{N}\left([S]_{N}\right), \cdots, S_{0} \prod_{i=1}^{N-k}\left(1-a_{i}\right)=\tau g_{N-k}\left([S]_{N}\right), \cdots, \\
& S_{0} \prod_{i=1}^{N-k-1}\left(1-a_{i}\right)=\tau g_{N-k-1}\left([S]_{N}\right), \cdots, S_{0}\left(1-a_{1}\right)=\tau g_{1}\left([S]_{N}\right),
\end{aligned}
$$

where $\tau>0$. Taking into account the relations (138) we obtain

$$
S_{0}\left(1-a_{1}\right)=\tau g_{1}\left([S]_{N}\right),
$$




$$
\tau g_{N-k-1}\left([S]_{N}\right)\left(1-a_{N-k}\right)=\tau g_{N-k}\left([S]_{N}\right), \quad k=\overline{2, N} .
$$

Solving the relations (139), we have

$$
a_{1}=1-\frac{\tau}{S_{0}} g_{1}\left([S]_{N}\right), \quad a_{N-k}=1-\frac{g_{N-k}\left([S]_{N}\right)}{g_{N-k-1}\left([S]_{N}\right)}, \quad k=\overline{2, N} .
$$

It is evident that $a_{N-k} \geq 0, k=\overline{2, N}$. To provide the positiveness of $a_{1}$ and the inequalities $\tau g_{N-n}\left([S]_{N}\right) \leq S_{N-n}, n=\overline{0, N-1}, S_{0} \geq S_{(0)}$, meaning that the random process (123) takes all the values from the sample $S_{n}, n=\overline{0, N}$, we must to put $\tau=\tau_{0} S_{(0)}, 0<\tau_{0} \leq 1$. It is evident that if $\tau_{0}=0$ then $a_{i}=1, i=\overline{1, N}$ Theorem 17 is proved.

Remark 4. It is evident that

$$
\begin{gathered}
a_{i}=1, i=\overline{N-k, N}, 1<k \leq N-1, a_{i}=1-\frac{g_{i}\left([S]_{N}\right)}{g_{i-1}\left([S]_{N}\right)}, i=\overline{2, N-k-1}, \\
a_{1}=1-\frac{\tau_{0} S_{(0)}}{S_{0}} g_{1}\left([S]_{N}\right), \quad 0<\tau_{0} \leq 1,
\end{gathered}
$$

is also estimation of the parameters $a_{1}, \cdots, a_{N}$ if

$$
0<g_{N-k-1}\left([S]_{N}\right) \leq g_{N-k-2}\left([S]_{N}\right) \leq \cdots \leq g_{1}\left([S]_{N}\right),[S]_{N} \in[0,1]^{N} .
$$

Such estimation is not interesting since

$$
\prod_{i=1}^{N-i}\left(1-a_{i}\right)=0, \quad i=\overline{0, k}
$$

Remark 5. If

$$
\begin{gathered}
g(x)= \begin{cases}\frac{S_{0}}{S_{(0)}} x, & \text { if } 0 \leq x \leq \frac{S_{(0)}}{S_{0}}, \\
1, & \text { if } \frac{S_{(0)}}{S_{0}}<x \leq 1,\end{cases} \\
g_{i}\left([S]_{N}\right)=g\left(\frac{S_{(N-i)}}{S_{(N)}}\right), \quad i=\overline{1, N}, \tau_{0}=1,
\end{gathered}
$$

then for the parameters $a_{1}, \cdots, a_{N}$ the estimation

$$
\begin{aligned}
& a_{i}= \begin{cases}1-\frac{S_{(N-i)}}{S_{(N-i+1)}}, & \text { if } \frac{S_{(N-i+1)}}{S_{(N)}} \leq \frac{S_{(0)}}{S_{0}}, \\
1-\frac{S_{(N-i)}}{S_{(N)}} \frac{S_{0}}{S_{(0)}}, & \text { if } \frac{S_{(N-i+1)}}{S_{(N)}}>\frac{S_{(0)}}{S_{0}}, \frac{S_{(N-i)}}{S_{(N)}} \leq \frac{S_{(0)}}{S_{0}}, \quad i=\overline{2, N}, \\
0, & \text { if } \frac{S_{(N-i)}}{S_{(N)}}>\frac{S_{(0)}}{S_{0}} .\end{cases} \\
& a_{1}= \begin{cases}1-\frac{S_{(N-1)}}{S_{(N)}}, & \text { if } \frac{S_{(N-1)}}{S_{(N)}} \leq \frac{S_{(0)}}{S_{0}}, \\
1-\frac{S_{(0)}}{S_{0}}, & \text { if } \frac{S_{(N-1)}}{S_{(N)}}>\frac{S_{(0)}}{S_{0}}\end{cases}
\end{aligned}
$$


is true. The following equalities

$$
\begin{aligned}
& \prod_{i=1}^{N}\left(1-a_{i}\right)=\frac{S_{(0)}}{S_{0}} g\left(\frac{S_{(0)}}{S_{(N)}}\right)=\frac{S_{(0)}}{S_{(N)}}, \\
& \prod_{i=1}^{N-k}\left(1-a_{i}\right)=\left\{\begin{array}{ll}
\frac{S_{(k)}}{S_{(N)}}, & \text { if } \frac{S_{(k)}}{S_{(N)}} \leq \frac{S_{(0)}}{S_{0}}, \\
\frac{S_{(0)}}{S_{0}}, & \text { if } \frac{S_{(k)}}{S_{(N)}}>\frac{S_{(0)}}{S_{0}},
\end{array} \quad k=\overline{1, N-1,},\right.
\end{aligned}
$$

are valid.

Remark 6. Suppose that $g(x)=x, x \in[0,1]$. Let us put

$$
\begin{gathered}
g_{N-i}\left([S]_{N}\right)=g\left(\frac{S_{(i)}}{S_{(N)}}\right)=\frac{S_{(i)}}{S_{(N)}}, i=\overline{0, k}, \quad g_{N-i}\left([S]_{N}\right)=1, \quad i=\overline{k+1, N-1} . \text { Then } \\
a_{1}=1-\tau_{0} \frac{S_{(0)}}{S_{0}}, \quad 0<\tau_{0} \leq 1, \quad a_{i}=0, \quad i=\overline{2, N-k-1}, \\
a_{i}=1-\frac{g_{i}\left([S]_{N}\right)}{g_{i-1}\left([S]_{N}\right)}, \quad i=\overline{N-k, N},
\end{gathered}
$$

is an estimation for the parameters $a_{1}, \cdots, a_{N}$.

In the next Theorems we put $\tau_{0}=1$. This corresponds to the fact that fair price of super-hedge is minimal for the considered statistic.

Theorem 18. On the measurable space $\{\Omega, \mathcal{F}\}$ with the filtration $\mathcal{F}_{n}$, $n=\overline{0, N}$, on it, let the discount risk asset evolution is given by the formula (123) with parameters $a_{i}, i=\overline{1, N}$, given by the formula (137). For the payoff function $f(x)=(x-K)^{+}, x \in(0, \infty), K>0$, the fair price of super-hedge is given by the formula

$$
\sup _{Q \in M_{0}^{N}} E^{Q} f\left(S_{N}\right)= \begin{cases}\left(S_{0}-K\right)^{+}, & \text {if } S_{(0)} g_{N}\left([S]_{N}\right) \geq K, \\ S_{0}\left(1-\frac{S_{(0)} g_{N}\left([S]_{N}\right)}{S_{0}}\right), & \text { if } S_{(0)} g_{N}\left([S]_{N}\right)<K .\end{cases}
$$

If $S_{(0)} g_{N}\left([S]_{N}\right) \geq K$, then the set of non arbitrage prices coincides with the point $\left(S_{0}-K\right)^{+}$, in case if $S_{(0)} g_{N}\left([S]_{N}\right)<K$ the set of non arbitrage prices coincides with the closed set $\left[\left(S_{0}-K\right)^{+}, S_{0}\left(1-\frac{S_{(0)} g_{N}\left([S]_{N}\right)}{S_{0}}\right)\right]$.

The fair price of super-hedge for the statistic (143), (144) is given by the formula

$$
\sup _{Q \in M_{0}^{N}} E^{Q} f\left(S_{N}\right)= \begin{cases}\left(S_{0}-K\right)^{+}, & \text {if } S_{0} \frac{S_{(0)}}{S_{(N)}} \geq K, \\ S_{0}\left(1-\frac{S_{(0)}}{S_{(N)}}\right), & \text { if } S_{0} \frac{S_{(0)}}{S_{(N)}}<K .\end{cases}
$$


If $S_{0} \frac{S_{(0)}}{S_{(N)}} \geq K$, then the set of non arbitrage prices coincides with the point $\left(S_{0}-K\right)^{+}$, in case if $S_{0} \frac{S_{(0)}}{S_{(N)}}<K$ the set of non arbitrage prices coincides with the closed set $\left[\left(S_{0}-K\right)^{+}, S_{0}\left(1-\frac{S_{(0)}}{S_{(N)}}\right)\right]$.

The fair price of super-hedge is minimal one for the statistic (137) with $g_{i}\left(X_{N}\right)=g_{N}\left(X_{N}\right)=1, \quad i=\overline{1, N-1}$, and is given by the formula

$$
\sup _{Q \in M_{0}^{N}} E^{Q} f\left(S_{N}\right)= \begin{cases}\left(S_{0}-K\right)^{+}, & \text {if } S_{(0)} \geq K, \\ S_{0}-S_{(0)}, & \text { if } S_{(0)}<K .\end{cases}
$$

If $S_{(0)} \geq K$, then the set of non arbitrage prices coincides with the point $\left(S_{0}-K\right)^{+}$, in case if $S_{(0)}<K$ the set of non arbitrage prices coincides with the closed set $\left[\left(S_{0}-K\right)^{+}, S_{0}-S_{(0)}\right]$.

Theorem 19. On the measurable space $\{\Omega, \mathcal{F}\}$ with the filtration $\mathcal{F}_{n}$, $n=\overline{0, N}$, on it, let the discount risk asset evolution is given by the formula (123), with the parameters $a_{i}, i=\overline{1, N}$, given by the formula (137). For the payoff function $f_{1}(x)=(K-x)^{+}, x \in(0, \infty), K>0$, the fair price of super-hedge is given by the formula

$$
\sup _{Q \in M_{0}^{N}} E^{Q} f_{1}\left(S_{N}\right)=f_{1}\left(S_{(0)} g_{N}\left([S]_{N}\right)\right) .
$$

The set of non arbitrage prices coincides with the closed interval

$$
\left[\left(K-S_{0}\right)^{+}, f_{1}\left(S_{(0)} g_{N}\left([S]_{N}\right)\right)\right] \text {. }
$$

The fair price of super-hedge for the statistic (143), (144) is given by the formula

$$
\sup _{Q \in M_{0}^{N}} E^{Q} f_{1}\left(S_{N}\right)=f_{1}\left(S_{0} \frac{S_{(0)}}{S_{(N)}}\right) .
$$

The set of non arbitrage prices coincides with the closed interval $\left[\left(K-S_{0}\right)^{+}, f_{1}\left(S_{0} \frac{S_{(0)}}{S_{(N)}}\right)\right]$.

The fair price of super-hedge is minimal one for the statistic (137) with $g_{i}\left(X_{N}\right)=g_{N}\left(X_{N}\right)=1, i=\overline{1, N-1}$, and is given by the formula

$$
\sup _{Q \in M_{0}^{N}} E^{Q} f_{1}\left(S_{N}\right)=f_{1}\left(S_{(0)}\right) \text {. }
$$

The set of non arbitrage prices coincides with the closed interval $\left[\left(K-S_{0}\right)^{+}, f_{1}\left(S_{(0)}\right)\right]$.

Theorem 20. On the measurable space $\{\Omega, \mathcal{F}\}$ with the filtration $\mathcal{F}_{n}$, $n=\overline{0, N}$, on it, let the discount risk asset evolution is given by the formula (123), with the parameters $a_{i}, i=\overline{1, N}$, given by the formula (137). For the payoff 
function $f_{1}\left(S_{0}, S_{1}, \cdots, S_{N}\right)=\left(K-\frac{\sum_{i=0}^{N} S_{i}}{N+1}\right)^{+}, K>0$, the fair price of super-hedge is given by the formula

$$
\sup _{Q \in M_{0}^{N}} E^{Q} f_{1}\left(S_{0}, S_{1}, \cdots, S_{N}\right)=\left(K-\frac{S_{0}+S_{(0)} \sum_{i=1}^{N} g_{i}\left([S]_{N}\right)}{N+1}\right)^{+} .
$$

The set of non arbitrage prices coincides with the closed interval

$$
\left[\left(K-S_{0}\right)^{+},\left(K-\frac{S_{0}+S_{(0)} \sum_{i=1}^{N} g_{i}\left([S]_{N}\right)}{N+1}\right)^{+}\right] \text {, if } K>\frac{S_{0}+S_{(0)} \sum_{i=1}^{N} g_{i}\left([S]_{N}\right)}{N+1} \text {. }
$$

For $K \leq \frac{S_{0}+S_{(0)} \sum_{i=1}^{N} g_{i}\left([S]_{N}\right)}{N+1}$ the set of non arbitrage prices coincides with the point 0 .

The fair price of super-hedge is minimal one for the statistic (137) with $g_{i}\left(X_{N}\right)=g_{N}\left(X_{N}\right)=1, i=\overline{1, N-1}$, and is given by the formula

$$
\sup _{Q \in M_{0}^{N}} E^{Q} f_{1}\left(S_{0}, S_{1}, \cdots, S_{N}\right)=\left(K-\frac{S_{0}+S_{(0)} N}{N+1}\right)^{+} .
$$

The set of non arbitrage prices coincides with the closed interval $\left[\left(K-S_{0}\right)^{+},\left(K-\frac{S_{0}+S_{(0)} N}{N+1}\right)^{+}\right]$, if $K>\frac{S_{0}+S_{(0)} N}{N+1}$. For $K \leq \frac{S_{0}+S_{(0)} N}{N+1}$ the set of non arbitrage prices coincides with the point 0 .

Theorem 21. On the measurable space $\{\Omega, \mathcal{F}\}$ with the filtration $\mathcal{F}_{n}$, $n=\overline{0, N}$, on it, let the discount risk asset evolution is given by the formula (123), with the parameters $a_{i}, i=\overline{1, N}$, given by the formula (137). For the payoff function $f\left(S_{0}, S_{1}, \cdots, S_{N}\right)=\left(\frac{\sum_{i=0}^{N} S_{i}}{N+1}-K\right)^{+}, K>0$, the fair price of super-hedge is given by the formula

$$
\begin{aligned}
& \sup _{Q \in M_{0}^{N}} E^{Q} f\left(S_{0}, S_{1}, \cdots, S_{N}\right) \\
& = \begin{cases}\left(S_{0}-K\right)^{+}, & \text {if } \frac{S_{0}+S_{(0)} \sum_{i=1}^{N} g_{i}\left([S]_{N}\right)}{N+1} \geq K, \\
\left(\begin{array}{c}
S_{0}+S_{(0)} \sum_{i=1}^{N} g_{i}\left([S]_{N}\right) \\
N+1
\end{array}\right. & \text { if } \frac{S_{0}+S_{(0)} \sum_{i=1}^{N} g_{i}\left([S]_{N}\right)}{N+1}<K .\end{cases}
\end{aligned}
$$


If $\frac{S_{0}+S_{(0)} \sum_{i=1}^{N} g_{i}\left([S]_{N}\right)}{N+1} \geq K$, then the set of non arbitrage prices coincides with the point $\left(S_{0}-K\right)^{+}$, in case if $\frac{S_{0}+S_{(0)} \sum_{i=1}^{N} g_{i}\left([S]_{N}\right)}{N+1}<K$ the set of non arbitrage prices coincides with the closed interval $\left[\left(S_{0}-K\right)^{+},\left(S_{0}-\frac{S_{0}+S_{(0)} \sum_{i=1}^{N} g_{i}\left([S]_{N}\right)}{N+1}\right)\right]$.

The fair price of super-hedge is minimal one for the statistic (137) with $g_{i}\left(X_{N}\right)=g_{N}\left(X_{N}\right)=1, i=\overline{1, N-1}$, and is given by the formula

$$
\begin{aligned}
& \sup _{Q \in M_{0}^{N}} E^{Q} f\left(S_{0}, S_{1}, \cdots, S_{N}\right) \\
& = \begin{cases}\left(S_{0}-K\right)^{+}, & \text {if } \frac{S_{0}+S_{(0)} N}{N+1} \geq K, \\
\left(S_{0}-\frac{S_{0}+S_{(0)} N}{(N+1)}\right), & \text { if } \frac{S_{0}+S_{(0)} N}{N+1}<K .\end{cases}
\end{aligned}
$$

If $\frac{S_{0}+S_{(0)} N}{N+1} \geq K$, then the set of non arbitrage prices coincides with the point $\left(S_{0}-K\right)^{+}$, in case if $\frac{S_{0}+S_{(0)} N}{N+1}<K$ the set of non arbitrage prices coincides with the closed interval $\left[\left(S_{0}-K\right)^{+},\left(S_{0}-\frac{S_{0}+S_{(0)} N}{N+1}\right)\right]$.

To estimate the parameters $\sigma, \mu$, let us define the likelihood function of the sample by the formula

$$
L(\sigma, \mu)=\frac{1}{\sigma^{N}} \exp \left\{-\frac{\sum_{i=1}^{N}\left(D_{i}\left(S_{i-1}, S_{i}\right)-d\right)^{2}}{2 \sigma^{2} \Delta t}\right\}
$$

using the formula (133) for $0<a_{i} \leq 1, i=\overline{1, N}$, where

$$
D_{i}\left(S_{i-1}, S_{i}\right)=\ln \left(\varphi_{i}\left(S_{i-1}, S_{i}\right) \frac{S_{i}-S_{i-1}\left(1-a_{i}\right)}{S_{i-1} a_{i}}\right) .
$$

Lemma 2. The maximum likelihood estimates of $\sigma, \mu$ are given by the formulas

$$
\begin{gathered}
\sigma^{2}=\frac{1}{N \Delta t} \sum_{i=1}^{N}\left(D_{i}\left(S_{i-1}, S_{i}\right)-\frac{1}{N} \sum_{i=1}^{N} D_{i}\left(S_{i-1}, S_{i}\right)\right)^{2}, \\
\mu=\frac{\sigma^{2}}{2}+r+\frac{1}{N \Delta t} \sum_{i=1}^{N} D_{i}\left(S_{i-1}, S_{i}\right) .
\end{gathered}
$$

Proof. For obtaining the estimates of $\sigma, \mu$, we use the Method of Maximum Likelihood. Let us calculate 


$$
\begin{aligned}
& \frac{\partial L(\sigma, \mu)}{\partial \sigma}=-\frac{N L(\sigma, \mu)}{\sigma}-L(\sigma, \mu) \frac{\partial}{\partial \sigma}\left[\frac{\sum_{i=1}^{N}\left(D_{i}\left(S_{i-1}, S_{i}\right)-d\right)^{2}}{2 \sigma^{2} \Delta t}\right] . \\
& \frac{\partial}{\partial \sigma}\left[\frac{\sum_{i=1}^{N}\left(D_{i}\left(S_{i-1}, S_{i}\right)-d\right)^{2}}{2 \sigma^{2} \Delta t}\right] \\
& =-\frac{1}{\sigma^{3} \Delta t} \sum_{i=1}^{N}\left(D_{i}\left(S_{i-1}, S_{i}\right)-d\right)^{2}-\frac{1}{\sigma^{2} \Delta t} \sum_{i=1}^{N}\left(D_{i}\left(S_{i-1}, S_{i}\right)-d\right) \frac{\partial d}{\partial \sigma} \\
& =-\frac{1}{\sigma^{3} \Delta t} \sum_{i=1}^{N}\left(D_{i}\left(S_{i-1}, S_{i}\right)-d\right)^{2}+\frac{1}{\sigma} \sum_{i=1}^{N}\left(D_{i}\left(S_{i-1}, S_{i}\right)-d\right) .
\end{aligned}
$$

Therefore

$$
\begin{gathered}
\frac{\partial L(\sigma, \mu)}{\partial \sigma}=-\frac{N L(\sigma, \mu)}{\sigma}-L(\sigma, \mu)\left[-\frac{1}{\sigma^{3} \Delta t} \sum_{i=1}^{N}\left(D_{i}\left(S_{i-1}, S_{i}\right)-d\right)^{2}\right. \\
\left.+\frac{1}{\sigma} \sum_{i=1}^{N}\left(D_{i}\left(S_{i-1}, S_{i}\right)-d\right)\right], \\
\frac{\partial L(\sigma, \mu)}{\partial \mu}=\frac{L(\sigma, \mu)}{\sigma} \sum_{i=1}^{N}\left(D_{i}\left(S_{i-1}, S_{i}\right)-d\right) .
\end{gathered}
$$

The maximum likelihood estimates for $\sigma$ and $\mu$ we can obtain from the equations

$$
\begin{gathered}
-\frac{N L(\sigma, \mu)}{\sigma}-L(\sigma, \mu)\left[-\frac{1}{\sigma^{3} \Delta t} \sum_{i=1}^{N}\left(D_{i}\left(S_{i-1}, S_{i}\right)-d\right)^{2}\right. \\
\left.+\frac{1}{\sigma} \sum_{i=1}^{N}\left(D_{i}\left(S_{i-1}, S_{i}\right)-d\right)\right]=0 \\
\frac{L(\sigma, \mu)}{\sigma} \sum_{i=1}^{N}\left(D_{i}\left(S_{i-1}, S_{i}\right)-d\right)=0 .
\end{gathered}
$$

Solving the equations (163), (164) we obtain the statement of Lemma 2.

Remark 7. If for some sample $S_{0}, S_{1}, \cdots, S_{N}$ the estimate of parameters $a_{1}, \cdots, a_{N}$ is such that $a_{i_{1}}=0, \cdots, a_{i_{k}}=0$ then in the estimates of $\sigma, \mu$ it is necessary to put $D_{i_{s}}\left(S_{i_{s}-1}, S_{i_{s}}\right)=0, s=\overline{1, k}$. This follows from the structure of random process (123), the transition probability function (126), the structure of the joint probability function (134) after Theorem 16.

\section{Conclusions}

In the paper, we generalize the results of the paper [1] [17]. In Section 2, we generalize an evolution of risk asset with memory proposed in the paper [1]. In Theorem 1, we describe completely the set of martingale measures for the considered evolution and prove that every martingale measure of this family is an integral over some measure on the set of extreme points of the set of martingale measures. In Theorem 3 the bound for every nonnegative $\mathcal{F}_{n}$ measurable random value the mathematical expectation for which relative to every martingale 
measure is bounded by 1 is found. In Theorem 4, it is proved that every nonnegative super-martingale relative to the regular set of measures is a local regular one. The same statement, as in Theorem 4, it is proved in Theorem 5 in the case, as a super-martingale is bounded from below.

Section 3 contains the application of the results obtained above to calculation of the interval of non-arbitrage prices for the wide class of evolutions of risky assets and payoff functions.

In Theorem 7, with the general assumptions about payoff functions and the evolution of risky assets, we found the non-arbitrage price interval and set the price of super-hedge. This set of payoff functions contains a payoff function of a standard European-type call option. Theorem 8 contains sufficient conditions regarding the evolution of risky assets (2) for which an interval of non-arbitrage prices has been found for a wide class of payoff functions. This class contains the payoff function for standard option put of European type.

Section 4 contains the results about the interval of non-arbitrage prices for the class of evolutions of risky assets described by the random process with parameters built on the geometric Brownian motion and payoff functions for call and put options of standard type.

In Theorem 9, a formula for the fair price of super-hedge is found for the evolution of risky assets given by the formula (89). Theorem 10 contains the estimates for the value of a super-hedge for a particular class of payoff functions including a payoff function for a standard call option.

In Theorem 11, the estimates for the value of a super-hedge for a particular class of payoff functions are found. This class of payoff functions includes a standard put option payoff function.

Theorem 12 gives the interval for non-arbitrage prices and the price of super-hedge in the case of a standard call options. The peculiarity of this formula is that the price of a super-hedge is proportional to the price of the underlying asset with a ratio less than one.

In Theorem 13, the formula for the fair price of super-hedge is found for put option of standard type.

Theorems 14, 15 contains the analogous results as in Theorems 12, 13 for the payoff functions of Asian type options.

Theorems 18, 19, 20, 21 of Section 5 are reformulations of Theorems 12, 13, 14,15 with taking into account the estimations of the parameters $a_{i}, i=\overline{1, N}$.

\section{Funding}

This work was partially supported by the Program of Fundamental Research of the Department of Physics and Astronomy of the National Academy of Sciences of Ukraine (project No. 0117U000240).

\section{Conflicts of Interest}

The author declares no conflicts of interest regarding the publication of this paper. 


\section{References}

[1] Gonchar, N.S. (2019) Description of Incomplete Financial Markets for Time Evolution of Risk Assets. Advances in Pure Mathematics, 9, 567-610. https://doi.org/10.4236/apm.2019.96029

[2] Eberlein, E. and Jacod, J. (1997) On the Range of Option Price. Finance and Stochastics, 1, 131-140. https://doi.org/10.1007/s007800050019

[3] Bellamy, N. and Jeanblanc, M. (1999) Incompleteness of Markets Driven by Mixed Diffusion. Finance and Stochastics, 4, 209-222. https://doi.org/10.1007/s007800050012

[4] Gonchar, N.S. (2008) Mathematical Foundations of Information Economics. Bogolyubov Institute for Theoretical Physics, Kiev.

[5] Gonchar, N.S. (2015) Mathematical Model of Banking Operation. Cybernetics and System Analysis, 51, 378-399. https://doi.org/10.1007/s10559-015-9730-0

[6] Gonchar, N.S. and Terentieva, L.S. (2008) Default Risk Valuation of the Firm with the Special Process of Internal Yield. Journal of Automation and Information Sciences, 40, 57-71. https://doi.org/10.1615/JAutomatInfScien.v40.i8.60

[7] Gonchar, N.S. (2017) Banking and Risk Assessment. In: Jerzy, K., Ed., Banking: Services, Opportunity and Risks, Nova Science Publisher, Inc., New York, Chapter 8.

[8] Kreps, D.M. (1981) Arbitrage and Equilibrium in Economics with Infinitely Many Commodities. Journal of Mathematical Economics, 8, 15-35.

https://doi.org/10.1016/0304-4068(81)90010-0

[9] Harrison, J.M. and Kreps, D.M. (1979) Martingales and Arbitrage in Multiperiod Securities Markets. Journal of Economic Theory, 20, 381-408. https://doi.org/10.1016/0022-0531(79)90043-7

[10] Harrison, J.M. and Pliska, S.R. (1981) Martingales and Stochastic Integrals in the Theory of Continous Trading. Stochastic Processes and their Applications, 11, 215260. https://doi.org/10.1016/0304-4149(81)90026-0

[11] Delbaen, F. and Schachermayer, W. (2006) The Mathematics and Arbitrage. Springer, Berlin.

[12] Dalang, R.C., Morton, A. and Willinger, W. (1990) Equivalent Martingale Measures and No-Arbitrage in Stochastic Securities Market Model. Stochastics and Stochastic Reports, 29, 185-201. https://doi.org/10.1080/17442509008833613

[13] Kramkov, D.O. (1996) Optional Decomposition of Super-Martingales and Hedging in Incomplete Security Markets. Probability Theory and Related Fields, 105, 459479. https://doi.org/10.1007/BF01191909

[14] Follmer, H. and Kramkov, D.O. (1997) Optional Decomposition Theorem under Constraints. Probability Theory and Related Fields, 109, 1-25. https://doi.org/10.1007/s004400050122

[15] Follmer, H. and Kabanov, Yu.M. (1996) Optional Decomposition Theorems in Discrete Time. Atti del convegno in onore di Oliviero Lessi, Padova, 25-26 marzo 1996, 47-68.

[16] Follmer, H. and Kabanov, Yu.M. (1998) Optional Decomposition and Lagrange Multipliers. Finance and Stochastics, 2, 69-81. https://doi.org/10.1007/s007800050033

[17] Gonchar, N.S. (2018) Martingales and Super-Martingales Relative to a Convex Set of Equivalent Measures. Advances in Pure Mathematics, 8, 428-462. https://doi.org/10.4236/apm.2018.84025

[18] Elliott, R. (1982) Stochastic Calculus and Applications. Springer, Berlin. 